\title{
CONCEPTIONS OF TOPOLOGICAL TRANSITIVITY ON SYMMETRIC PRODUCTS
}

\author{
Franco Barragán ${ }^{1, a}$, Sergio Macías ${ }^{2, b}$ and Anahí Rojas ${ }^{1, *}$ \\ ${ }^{1}$ Instituto de Física y Matemáticas, Universidad Tecnológica de la Mixteca, Carretera a Acatlima, Km. 2.5, \\ Huajuapan de León, Oaxaca, C.P. 69000, México \\ a franco@mixteco.utm.mx \\ ${ }^{2}$ Instituto de Matemáticas, Universidad Nacional Autónoma de México, Circuito Exterior, Ciudad Universitaria, \\ Ciudad de México, CDMX, C.P. 04510, México \\ ${ }^{b}$ sergiom@matem.unam.mx \\ Communicated by László Tóth \\ Original Research Paper \\ Received: Mar 12, 2019 • Accepted: Feb 17, 2020 \\ First published online: Mar 24, 2021 \\ ๑) 2020 The Author(s)
}

\section{ABSTRACT}

Let $X$ be a topological space. For any positive integer $n$, we consider the $n$-fold symmetric product of $X, \mathcal{F}_{n}(X)$, consisting of all nonempty subsets of $X$ with at most $n$ points; and for a given function $f: X \rightarrow X$, we consider the induced functions $\mathcal{F}_{n}(f): \mathcal{F}_{n}(X) \rightarrow \mathcal{F}_{n}(X)$. Let $\mathcal{M}$ be one of the following classes of functions: exact, transitive, $\mathbb{Z}$-transitive, $\mathbb{Z}_{+}$-transitive, mixing, weakly mixing, chaotic, turbulent, strongly transitive, totally transitive, orbit-transitive, strictly orbit-transitive, $\omega$-transitive, minimal, $I N, T T_{++}$, semi-open and irreducible. In this paper we study the relationship between the following statements: $f \in \mathcal{M}$ and $\mathcal{F}_{n}(f) \in \mathcal{M}$.

\section{KEYWORDS}

topological transitivity, symmetric products, dynamical systems

\section{MATHEMATICS SUBJECT CLASSIFICATION (2020)}

Primary 54B20; Secondary 37B02, 37B45, 54F15

\section{INTRODUCTION}

In the last 30 years, discrete dynamical systems had been greatly developed. This is because they are very useful to model problems of other Sciences such as: Chemistry, Physics, Biology and Economics. In the literature we can find several types of dynamical systems: exact, mixing, weakly mixing, transitive, totally transitive, strongly transitive, chaotic, minimal and irreducible. Several of these systems are introduced for metric spaces and have been extensively studied, see [1], [5], [7], [10], [21], [26], [25], [27], [30] and [40].

In particular, transitivity is one of the most important properties in topological dynamics, it was introduced in 1920 by G. D. Birkhoff [10], for metric spaces. Since then, this concept has been studied extensively. This notion has been generalized to topological spaces. In addition, other

${ }^{*}$ Corresponding author. E-mail: anacarrasco.rr@gmail.com 
very similar definitions have been given, which are related or are equivalent to the transitivity in topological spaces with particular properties, namely: orbit-transitivity, strictly orbit-transitivity and $\omega$-transitivity, see [2] and [34]. Also, in [9], the notions of $\mathbb{N}$-transitivity, $\mathbb{N}_{0}$-transitivity and $\mathbb{Z}$-transitivity are introduced.

With respect to the nonmetric case, Gottschalk and Hedlund [19] study the dynamics of transformation groups in uniform spaces. The authors of [12] and [13] consider notions of expansion in the class of uniform spaces. Hood in [22] defines entropy for uniform spaces. In [3] the authors generalize many known results about equicontinuity to the uniform world and in [18] several concepts are defined on Hausdorff spaces or Tychonoff spaces, for example, the authors prove that in a Tychonoff space, transitivity and dense periodic points imply (uniform) sensitivity to initial conditions. They also study versions of shadowing, generalizing a number of well-known results to the topological setting.

On the other hand, the hyperspace theory had its beginnings in the early 1900's, with the works of F. Hausdorff and L. Vietoris. Given a compact metric space $X$, the hyperspaces of $X$ most studied are: the hyperspace $2^{X}$ which consists of all the nonempty compact subsets of $X$; given a natural number $n$, the hyperspace $\mathcal{C}_{n}(X)$ consisting of the elements of $2^{X}$ that have the most $n$ components (the $n$-fold hyperspace of $X$ ); and the hyperspace $\mathcal{F}_{n}(X)$ formed by the elements of $2^{X}$ which have at most $n$ points (the $n$-fold symmetric product of $X$ ). Each of them is topologized with the Hausdorff metric. These hyperspace are extensively studied in continuum theory [23], [33] and [24].

Given a compact metric space $X$ and a map (continuous function) $f: X \rightarrow X$, consider the induced function $2^{f}: 2^{X} \rightarrow 2^{X}$ such that $2^{f}(A)=f(A)$, for each $A \in 2^{X}$. Define $\mathcal{C}_{n}(f)=\left.2^{f}\right|_{\mathcal{C}_{n}(X)}$ and $\mathcal{F}_{n}(f)=\left.2^{f}\right|_{\mathcal{F}_{n}(X)}$. Thus, the dynamical system $(X, f)$ induces dynamical systems $\left(2^{X}, 2^{f}\right)$, $\left(\mathcal{C}_{n}(X), \mathcal{C}_{n}(f)\right)$ and $\left(\mathcal{F}_{n}(X), \mathcal{F}_{n}(f)\right)$. Note that we require the continuity of the function $f$ in order to have well defined induced function on $2^{X}$ and $\mathcal{C}_{n}(X)$. This is not the case for $\mathcal{F}_{n}(f)$, this is well defined for a not necessarily continuous function.

One line of research consists of analyze the relationships between the dynamical system $(X, f)$ and the dynamical systems $\left(2^{X}, 2^{f}\right),\left(\mathcal{C}_{n}(X), \mathcal{C}_{n}(f)\right)$ and $\left(\mathcal{F}_{n}(X), \mathcal{F}_{n}(f)\right)$. The first study of the induced dynamical systems on hyperspaces is given by Bauer and Sigmund in 1975 [8]. However, during the last quarter of the 20th century, the study of induced dynamics on hyperspaces was fairly inactive. In recent years, the research on hyperspace dynamics has been considered, for example: Román-Flores on transitivity [40]; J. Banks on Chaos [5]; Peris on mixing, weak mixing, transitivity and Auslander-Yorke chaos [39]; Liao et al. [30] on transitivity, mixing and chaos; Peña and López on entropy [37]; Kwietniak and Oprocha on entropy, mixing and weak mixing [27]; Wang et al. [44] on sensitivity; Lampart and Raith on entropy [28]; Li on stronger forms of sensitivity [29]; and Fernández et al. [14] on chain transitivity.

Particularly, the $n$-fold symmetric product of $X, \mathcal{F}_{n}(X)$, is introduced in 1931 by K. Bursuk and S. Ulam [11]. Several topological properties are studied, for example see [31], [32] and [35]. Other, researchers have studied dynamical properties of symmetric products, for example: Kwietniak and Misiurewicz on chaos [26]; Higuera and Illanes on transitivity, chaos, sensitivity and mixing [16]; Gómez-Rueda et al. [16] on periodicity, recurrence, quasi-periodicity, wandering points, shadowing and exactness; and Barragán et al. [7] on transitivity, chaocity, mixing, weak mixing, strongly transitivity, totally transitivity and irreducibility.

It is remarkable that there exists a variety of results about dynamical properties on hyperspaces, when $X$ is a continuum and $f$ a surjective map [7], [16] and [21]. Recently, in [17], C. Good and S. Macías investigated some topological properties of $n$-fold symmetric product $\mathcal{F}_{n}(X)$, when $X$ is a Hausdorff topological space. Liang-Xue Peng and Yuan Sun [38] and Tang et al. [42] also obtain information about the $n$-fold symmetric product of Hausdorff spaces.

In this paper we analyze the relationship that exists between the following dynamical systems $(X, f)$ and $\left(\mathcal{F}_{n}(X), \mathcal{F}_{n}(f)\right)$ when $X$ is a topological space. Specifically, if $\mathcal{M}$ is one of the following classes of functions: exact, transitive, $\mathbb{Z}$-transitive, $\mathbb{Z}_{+}$-transitive, mixing, weakly mixing, chaotic, turbulent, strongly transitive, totally transitive, orbit-transitive, strictly orbit-transitive, $\omega$-transitive, 
minimal, $I N, T T_{++}$, semi-open or irreducible, we study the relationships between the following statements:

(1) $f \in \mathcal{M}$.

(2) $\mathcal{F}_{n}(f) \in \mathcal{M}$.

The paper has six sections. In Section 2, we recall the basic definitions and give the notation required. In Section 3, we study the necessary topological properties of $n$-fold symmetric product that we need for the corresponding results of dynamical properties of the induced function $\mathcal{F}_{n}(f)$. In Section 4, we see the relationship between the functions $f$ and $\mathcal{F}_{n}(f)$, when one of these is: $\mathbb{Z}$ transitive, mixing, semi-open, exact, transitive, weakly mixing, totally transitive, strongly transitive, minimal, chaotic and turbulent. In Section 5, we analyze the problem given in Section 4, when one of the functions is: orbit-transitive, strictly orbit-transitive, $\omega$-transitive, $I N, T T_{++} \mathbb{Z}$-transitive and $\mathbb{Z}_{+}$-transitive. Finally, in Section 6, we investigate the problem of Section 5 giving conditions to the phase space $X$ and/or to the function $f$.

\section{DEFINITIONS AND NOTATIONS}

A set is said to be nondegenerate if it has more than one point. A (discrete) dynamical system is a pair $(X, f)$, where $X$ is a nondegenerate topological space and $f: X \rightarrow X$ is a function, $X$ is called the phase space. Let $X$ be a topological space and let $A$ be a subset of $X, \operatorname{cl}_{X}(A)$ and $\operatorname{int}_{X}(A)$ denote the closure and interior of the set $A$ in $X$, respectively. The symbols $\mathbb{Z}, \mathbb{Z}_{+}$and $\mathbb{N}$ denote the set of integers, the set of nonnegative integers and the set of positive integers, respectively. If $X$ is a topological space, the Cartesian product of $X$ with itself $n$ times is denoted by $X^{n}$.

Given a dynamical system $(X, f)$, for each $k \in \mathbb{N}$, the $k$ th iteration of $f$ is defined as the repeated composition of $f$ with itself $k$ times and is denoted by $f^{k}$. This is, $f^{k}=f \circ f^{k-1}$, where $f^{1}=f$ and $f^{0}=i d_{X}$, the identity function on $X$. For a subset $A$ of $X$ and $k \in \mathbb{Z}$, we denote by $f^{k}(A)$ the image of $A$ under $f^{k}$, where $k \geq 0$, and the preimage under $f^{|k|}$ where $k<0$. If $z \in X, f^{-k}(z)$ denotes the set $f^{-k}(\{z\})$, for each $k>0$.

Let $(X, f)$ be a dynamical system and let $x \in X$. The orbit of $x$ under $f$ is the $\operatorname{set} \mathcal{O}(x, f)=\left\{f^{k}(x) \mid\right.$ $\left.k \in \mathbb{Z}_{+}\right\}$. A point $x$ of $X$ is a transitive point of the function $f$ if the set $\mathcal{O}(x, f)$ is dense in $X$. The set of transitive points of $f$ is denoted by $\operatorname{trans}(f)$. The point $x$ is a fixed point of $f$ if $f(x)=x$. The point $x$ is a periodic point of $f$ if there exists $k \in \mathbb{N}$ such that $f^{k}(x)=x$. The set of periodic points of $f$ is denoted by $\operatorname{Per}(f)$. If $k=\min \left\{m \in \mathbb{N} \mid f^{m}(x)=x\right\}$, we say that $k$ is the period of $x$ under $f$. A point $y$ in $X$ is a $\omega$-limit point of $x$ under $f$ if for any $k \in \mathbb{N}$ and for any open subset $U$ of $X$ such that $y \in U$, there exists a positive integer $m \geq k$ such that $f^{m}(x) \in U$. The set of $\omega$-limit points of $x$ under $f$, is denoted by $\omega(x, f)$ and is called $\omega$-limit set of $x$. Given a subset $A$ of $X$, we say that $A$ is + invariant under $f$ if $f(A) \subseteq A, A$ is -invariant under $f$ if $f^{-1}(A) \subseteq A$ and $A$ is invariant under $f$ if $f(A)=A$. For subsets $A$ and $B$ of $X$, we define the following subsets of $\mathbb{Z}$ :

a) $N_{f}(A, B)=\left\{k \in \mathbb{Z} \mid A \cap f^{-k}(B) \neq \varnothing\right\}$,

b) $n_{f}(A, B)=\left\{k \in \mathbb{Z}_{+} \mid A \cap f^{-k}(B) \neq \varnothing\right\}$.

A topological space $X$ is pseudo-regular if for any nonempty open subset $U$ of $X$, there exists a nonempty open subset $V$ of $X$ such that $\operatorname{cl}_{X}(V) \subseteq U$ [34]. The topological space $X$ is partially compact and pseudo-regular if there exists a nonempty open subset $U$ of $X$ such that $\mathrm{cl}_{X}(U)$ is compact and pseudo-regular [34]. Let $X$ be a topological space, let $A$ be a subset of $X$ and let $a \in A$. We say that $a$ is an isolated point of $A$ if there exists an open subset $U$ of $X$ such that $U \cap A=\{x\}$. A point $x$ of $X$ is a quasi-isolated point of $X$ if there exists a dense subset $B$ of $X$ such that $x \in B$ and $x$ is an isolated point of $B$ [34]. A topological space is perfect if it does not have isolated points. The following definitions can be found in [2], [10] and [34].

Let $X$ be a topological space and let $f: X \rightarrow X$ be a function. Then $f$ is:

- Transitive, if for every pair of nonempty open subsets $U$ and $V$ of $X$, there exists $k \in \mathbb{N}$ such that $f^{k}(U) \cap V \neq \varnothing$ (equivalently, for each pair of nonempty open subsets $U$ and $V$ of $X$, $\left.n_{f}(U, V) \backslash\{0\} \neq \varnothing\right)$. 
- Chaotic, if it is transitive and $\operatorname{Per}(f)$ is dense in $X$.

- Exact, if for each nonempty open subset $U$ of $X$, there exists $k \in \mathbb{N}$ such that $f^{k}(U)=X$.

- IN, if $X$ is not the union of two proper, closed and +invariant subsets under $f$ of $X$.

- Irreducible, if the only closed subset $A$ of $X$ such that $f(A)=X$ is $X$.

- Minimal, if for each nonempty closed +invariant subset under $f A$ of $X$, we have that $A=X$.

- Mixing, if for each pair of nonempty open subsets $U$ and $V$ of $X$, there exists $N \in \mathbb{N}$ such that $f^{k}(U) \cap V \neq \varnothing$, for all $k \geq N$.

- $\omega$-transitive, if there exists $x \in X$ such that $\omega(x, f)=X$.

- Orbit-transitive, if there exists $x \in X$ such that $\operatorname{cl}_{X}(\mathcal{O}(x, f))=X$.

- Semi-open, if for each nonempty open subset $U$ of $X$, it follows that $\operatorname{int}_{X}(f(U)) \neq \varnothing$.

- Strictly orbit-transitive, if there exists a point $x$ in $X$ such that $\operatorname{cl}_{X}(\mathcal{O}(f(x), f))=X$.

- Strongly transitive, if for each nonempty open subset $U$ of $X$, there exists $s \in \mathbb{N}$ such that $X=\bigcup_{k=0}^{s} f^{k}(U)$.

- Totally transitive, if $f^{s}$ is transitive, for all $s \in \mathbb{N}$.

- $T T_{++}$, if for any pair of nonempty open subsets $U$ and $V$ of $X$, the set $n_{f}(U, V)$ is infinite.

- Turbulent, if there exist compact nondegenerate subsets $C$ and $K$ of $X$ such that $C \cap K$ has at most one point and $K \cup C \subseteq f(K) \cap f(C)$.

- Weakly mixing, if for any nonempty open subsets $U_{1}, U_{2}, V_{1}$ and $V_{2}$ of $X$, there exists $k \in \mathbb{N}$ such that $f^{k}\left(U_{i}\right) \cap V_{i} \neq \varnothing$, for each $i \in\{1,2\}$.

- $\mathbb{Z}$-transitive, if for each pairs of nonempty open subsets $U$ and $V$ of $X$, the subset $N_{f}(U, V) \neq \varnothing$.

- $\mathbb{Z}_{+}$-transitive, if for all pair of nonempty open subsets $U$ and $V$ of $X$, the set $n_{f}(U, V) \neq \varnothing$.

In the diagram of Figure 1, we put the inclusions between some of these classes of functions for the general case, this is when $X$ is a topological space and $f: X \rightarrow X$ is a function. For the proofs of these inclusions see [2], [9] and [34].

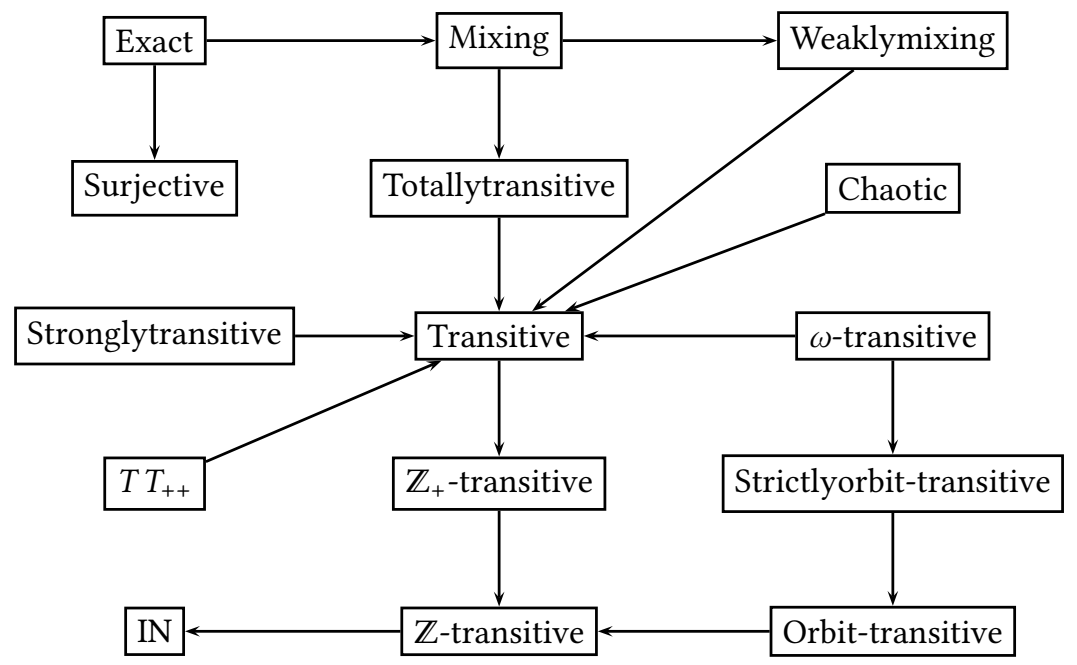

FIGURE 1. Relationships between functions

Given a topological space $X$ and a function $f: X \rightarrow X$, it is not difficult to verify that $f$ is minimal if $\operatorname{cl}_{X}(\mathcal{O}(x, f))=X$, for each $x \in X$.

When we add properties to the phase space or the function in Figure 1, we obtain other relationships, namely: Let $X$ be a topological space and let $f: X \rightarrow X$ be a function. If $f$ is a map (continuous function), it follows that: if $f$ is weakly mixing, then $f$ is totally transitive ([5, Theorem 1 (e)]); if $f$ is strictly orbit-transitive, then $f$ is $\omega$-transitive [34, Theorem 3.2]; $f$ is minimal if and only if $\operatorname{cl}_{X}(\mathcal{O}(x, f))=X$, for each $x \in X$. Also, if $X$ does not have quasi-isolated points, it follows that: (a) if $f$ is orbit-transitive, then $f$ is transitive [34, Theorem 4.1]; $(b)$ if $f$ is orbit-transitive, 
then $f$ is strictly orbit-transitive [34, Theorem 2.7]; and (c) if $f$ is strictly orbit-transitive, then $f$ is $\omega$-transitive [34, Theorem 3.2].

THEOREM 2.1. Let $X$ be a topological space, let $f: X \rightarrow X$ be a map and let $n \in \mathbb{N}$. Then the following are equivalent:

(1) $f$ is weakly mixing.

(2) For each $m \geq 2$ and for all nonempty open subsets $U_{1}, \ldots, U_{m}, V_{1}, \ldots, V_{m}$ of $X$, there exists $k \in \mathbb{N}$ such that $f^{k}\left(U_{i}\right) \cap V_{i} \neq \varnothing$, for each $i \in\{1,2, \ldots, m\}$.

(3) For any nonempty open subsets $U, V_{1}, V_{2}$ of $X$, there exists $k \in \mathbb{N}$ such that $f^{k}(U) \cap V_{i} \neq \varnothing$, for each $i \in\{1,2\}$.

Proof. Suppose that $f$ is weakly mixing. Let $U_{1}, \ldots, U_{m}, V_{1}, \ldots, V_{m}$ be nonempty open subsets of $X$. By [15, Proposition II.3], the product map $f^{\times m}: X^{m} \rightarrow X^{m}$ such that $f^{\times m}\left(x_{1}, \ldots, x_{m}\right)=\left(f\left(x_{1}\right), \ldots, f\left(x_{n}\right)\right)$ is transitive. Hence, there exists $k \in \mathbb{N}$ such that $f^{k}\left(U_{i}\right) \cap V_{i} \neq \varnothing$, for each $i \in\{1,2, \ldots, m\}$. Therefore, we have that (1) implies (2).

Suppose that for each $m \geq 2$ and for any nonempty open subsets $U_{1}, \ldots, U_{m}, V_{1}, \ldots, V_{m}$ of $X$, there exists $k \in \mathbb{N}$ such that $f^{k}\left(U_{i}\right) \cap V_{i} \neq \varnothing$, for each $i \in\{1,2, \ldots, m\}$. Let $U, V_{1}, V_{2}$ be nonempty open subsets of $X$. Then, by hypothesis, there exists $k \in \mathbb{N}$ such that $f^{k}(U) \cap V_{i} \neq \varnothing$, for each $i \in\{1,2\}$. Thus, we have that (2) implies (3).

The fact that (3) implies (1) is proven in [4, Lemma 5].

\section{SOME PROPERTIES ON SYMMETRIC PRODUCTS}

Hyperspaces theory started about 1900, with the work of F. Hausdorff [20] and L. Vietoris [43]. Nowadays hyperspaces are widely studied, mainly in continuum theory, see [23], [33] and [24]. We study dynamical properties of $n$-fold symmetric products, introduced at 1931 by K. Borsuk and S. Ulam [11].

Given a topological space $(X, \tau)$ and a positive integer $n$, we define the $n$-fold symmetric product of $X$ by:

$$
\mathcal{F}_{n}(X)=\{A \subseteq X \mid A \neq \varnothing \text { and } A \text { has at most } n \text { elements }\} \text {. }
$$

This hyperspace is considered with the Vietoris topology [24]. Next, we describe this topology.

Given a finite collection of nonempty subsets $U_{1}, \ldots, U_{m}$ of $X$, we denote by $\left\langle U_{1}, \ldots, U_{m}\right\rangle_{n}$ the subset of $\mathcal{F}_{n}(X)$ :

The family:

$$
\left\{A \in \mathcal{F}_{n}(X) \mid A \subseteq \bigcup_{i=1}^{m} U_{i} \text { and } A \cap U_{i} \neq \varnothing, \text { for each } i \in\{1, \ldots, m\}\right\} .
$$

$$
\mathcal{B}=\left\{\left\langle U_{1}, \ldots, U_{m}\right\rangle_{n} \mid U_{i} \in \tau, \text { for each } i \in\{1, \ldots, m\} \text { and } m \in \mathbb{N}\right\}
$$

forms a base for a topology on $\mathcal{F}_{n}(X)$, which is denoted by $\tau_{V}$, and called Vietoris topology.

The following theorem was proved in [21, Lemma 4.2].

THEOREM 3.1. Let $(X, \tau)$ be a topological space and let $n \in \mathbb{N}$. Then

$$
\beta^{\prime}=\left\{\left\langle U_{1}, \ldots, U_{n}\right\rangle_{n} \mid U_{i} \in \tau, \text { for each } i \in\{1, \ldots, n\}\right\}
$$

is a base for the Vietoris topology $\tau_{V}$ on $\mathcal{F}_{n}(X)$.

The following result is easy to establish [11, p. 877], [17, Lemma 2.8] and [23, p. 8].

THEOREM 3.2. Let $X$ be a topological space and let $n \in \mathbb{N}$. Then the following hold.

(1) $f_{n}: X^{n} \rightarrow \mathcal{F}_{n}(X)$ given by $f_{n}\left(\left(x_{1}, \ldots, x_{n}\right)\right)=\left\{x_{1}, \ldots, x_{n}\right\}$, for each $\left(x_{1}, \ldots, x_{n}\right) \in X^{n}$, is a map.

(2) If $A \in \mathcal{F}_{n}(X)$, then $f_{n}\left(A^{n}\right)=\mathcal{F}_{n}(A)$.

(3) If $U_{1}, \ldots, U_{n}$ are subsets of $X$, then:

$$
\operatorname{cl}_{F_{n}(X)}\left(\left\langle U_{1}, \ldots, U_{n}\right\rangle_{n}\right)=\left\langle\operatorname{cl}_{X}\left(U_{1}\right), \ldots, \operatorname{cl}_{X}\left(U_{n}\right)\right\rangle_{n} .
$$

(4) If $\mathcal{V}$ is an open subset of $\mathcal{F}_{n}(X)$, then $\bigcup \mathcal{V}$ is an open subset of $X$.

(5) If $A \subset X$ and $A$ is compact, then $\langle A\rangle_{n}$ is compact.

(6) If $A \subseteq X$ and $A$ is closed in $X$, then $\langle A\rangle_{n}$ is a closed subset in $\mathcal{F}_{n}(X)$. 
DEFINITION 3.3. Let $X$ be a topological space, let $f: X \rightarrow X$ be a function and let $n \in \mathbb{N}$. The function denoted by $\mathcal{F}_{n}(f): \mathcal{F}_{n}(X) \rightarrow \mathcal{F}_{n}(X)$ and defined by:

$$
\mathcal{F}_{n}(f)(A)=f(A), \text { for each } A \in \mathcal{F}_{n}(X)
$$

it is call induced function by $f$ to hyperspace $\mathcal{F}_{n}(X)$.

REMARK 3.4. Let $X$ be a topological space, let $f: X \rightarrow X$ be a function, and let $n \in \mathbb{N}$. It follows that the dynamical system $(X, f)$ induces the dynamical system $\left(\mathcal{F}_{n}(X), \mathcal{F}_{n}(f)\right)$.

THEOREM 3.5. Let $X$ be a topological space, let $f: X \rightarrow X$ be a function, let $F$ be a subset of $X$ and let $n \in \mathbb{N}$. If $F$ is +invariant under $f$ in $X$, then $\langle F\rangle_{n}$ is +invariant under $\mathcal{F}_{n}(f)$ in $\mathcal{F}_{n}(X)$.

Proof. Suppose that $F$ is +invariant under $f$. We prove that $\langle F\rangle_{n}$ is +invariant under $\mathcal{F}_{n}(f)$ in $\mathcal{F}_{n}(X)$. Let $A \in\langle F\rangle_{n}$. Then $A \subseteq F$ and $f(A) \subseteq f(F)$. Since $F$ is +invariant under $f, f(A) \subseteq F$. Hence, $f(A) \in\langle F\rangle_{n}$. Now, since $\mathcal{F}_{n}(f)(A)=f(A)$, we obtain that $\mathcal{F}_{n}(f)(A) \in\langle F\rangle_{n}$. Thus, $\mathcal{F}_{n}(f)\left(\langle F\rangle_{n}\right) \subseteq\langle F\rangle_{n}$. Therefore, $\langle F\rangle_{n}$ is +invariant under $\mathcal{F}_{n}(f)$ in $\mathcal{F}_{n}(X)$.

The following theorem shows that the continuity of the function in [21, Lemma 4.7] is not necessary.

THEOREM 3.6. Let $X$ be a topological space, let $f: X \rightarrow X$ be a function and let $n \in \mathbb{N}$. The set $\operatorname{Per}(f)$ is a dense subset in $X$ if and only if $\operatorname{Per}\left(\mathcal{F}_{n}(f)\right)$ is a dense subset in $\mathcal{F}_{n}(X)$.

Proof. Suppose that $\operatorname{Per}(f)$ is a dense subset in $X$. We prove that $\operatorname{Per}\left(\mathcal{F}_{n}(f)\right)$ is a dense subset in $\mathcal{F}_{n}(X)$. Let $\mathcal{V}$ be a nonempty open subset of $\mathcal{F}_{n}(X)$. Then, by Theorem 3.1, there exist nonempty open subsets $U_{1}, \ldots, U_{n}$ of $X$ such that $\left\langle U_{1}, \ldots, U_{n}\right\rangle_{n} \subseteq \mathcal{V}$. Since $\operatorname{Per}(f)$ is a dense subset of $X$, we have that, for each $i \in\{1, \ldots, n\}, \operatorname{Per}(f) \cap U_{i} \neq \varnothing$. For each $i \in\{1, \ldots, n\}$, let $x_{i} \in \operatorname{Per}(f) \cap U_{i}$. Then, for each $i \in\{1, \ldots, n\}$, there exists $k_{i} \in \mathbb{N}$ such that $f^{k_{i}}\left(x_{i}\right)=x_{i}$. Let $k=k_{1} \cdots k_{n}$. It follows that, for each $i \in\{1, \ldots, n\}, f^{k}\left(x_{i}\right)=x_{i}$. Let $A=\left\{x_{1}, \ldots, x_{n}\right\}$. Note that $A \in\left\langle U_{1}, \ldots, U_{n}\right\rangle_{n}$ and $\left[\mathcal{F}_{n}(f)\right]^{k}(A)=A$. Then $A \in \mathcal{U} \cap \operatorname{Per}\left(\mathcal{F}_{n}(f)\right)$. Thus, $\mathcal{V} \cap \operatorname{Per}\left(\mathcal{F}_{n}(f)\right) \neq \varnothing$. Therefore, $\operatorname{Per}\left(\mathcal{F}_{n}(f)\right)$ is a dense subset of $\mathcal{F}_{n}(X)$.

Now, suppose that $\operatorname{Per}\left(\mathcal{F}_{n}(f)\right)$ is a dense subset in $\mathcal{F}_{n}(X)$, we show that $\operatorname{Per}(f)$ is a dense subset of $X$. Let $U$ be a nonempty open subset of $X$. Then $\langle U\rangle_{n}$ is a nonempty open subset of $\mathcal{F}_{n}(X)$. Since $\operatorname{Per}\left(\mathcal{F}_{n}(f)\right)$ is dense in $\mathcal{F}_{n}(X)$, we have that $\operatorname{Per}\left(\mathcal{F}_{n}(f)\right) \cap\langle U\rangle_{n} \neq \varnothing$. Let $A=\left\{x_{1}, \ldots, x_{r}\right\} \in$ $\operatorname{Per}\left(\mathcal{F}_{n}(f)\right) \cap\langle U\rangle_{n}$. Then, there exists $k \in \mathbb{N}$ such that $\left[\mathcal{F}_{n}(f)\right]^{k}(A)=A$ and $A \subseteq U$. Hence, $f^{k}(A)=A$ and $A \subseteq U$. It follows that $\left.f^{k}\right|_{A}: A \rightarrow A$ is a permutation. Since $A$ is a finite subset, we have that the number of permutations of $A$ is $r$ !. Then there exists $m \in \mathbb{N}$ such that $\left.\left(f^{k}\right)^{m}\right|_{A}=i d_{A}$. Thus, for each $i \in\{1, \ldots, r\}, f^{k m}\left(x_{i}\right)=x_{i}$ and $x_{i} \in U$. Therefore, $U \cap \operatorname{Per}(f) \neq \varnothing$ and $\operatorname{Per}(f)$ is a dense subset in $X$.

The following results extends [38, Proposition 8] to arbitrary topological spaces.

THEOREM 3.7. Let $(X, \tau)$ be a topological space. Then $X$ has a countable base if and only if $\mathcal{F}_{n}(X)$ has a countable base.

Proof. Let $\gamma=\left\{V_{i} \in \tau \mid i \in \mathbb{N}\right\}$ be a countable base of $X$. We prove that:

$$
\gamma^{\prime}=\left\{\left\langle V_{1}, \ldots, V_{l}\right\rangle_{n} \mid V_{i} \in \gamma, \text { for each } i \in\{1, \ldots, l\} \text { and } l \in \mathbb{N}\right\}
$$

is a countable base of $\mathcal{F}_{n}(X)$. Clearly, $\gamma^{\prime}$ is a countable collection. By Theorem 3.1, $\beta^{\prime}=\left\{\left\langle U_{1}, \ldots, U_{n}\right\rangle_{n} \mid\right.$ $U_{i} \in \tau$, for each $\left.i \in\{1, \ldots, n\}\right\}$ forms a base for the Vietoris topology, $\tau_{V}$, on $\mathcal{F}_{n}(X)$. Hence, it is enough to verify that for every $\mathcal{A} \in \beta^{\prime}$ and for each $A \in \mathcal{A}$, there exist $V_{1}, \ldots, V_{l}$ in $\gamma$, with $l \in \mathbb{N}$, such that $A \in\left\langle V_{1}, \ldots, V_{l}\right\rangle_{n} \subseteq \mathcal{A}$. Let $\mathcal{A} \in \beta^{\prime}$ and let $A=\left\{x_{1}, \ldots, x_{k}\right\} \in \mathcal{A}$. By Theorem 3.1, there exist nonempty open subsets $U_{1}, \ldots, U_{n}$ of $X$ such that $A \in\left\langle U_{1}, \ldots, U_{n}\right\rangle_{n} \subseteq \mathcal{A}$. Let $U_{x_{i}}=\bigcap\left\{U \in\left\{U_{1}, \ldots, U_{n}\right\} \mid x_{i} \in\right.$ $U\}$, for each $i \in\{1, \ldots, k\}$. Note that $A \in\left\langle U_{x_{1}}, \ldots, U_{x_{k}}\right\rangle_{n} \subseteq\left\langle U_{1}, \ldots, U_{n}\right\rangle_{n}$. By hypothesis, for each $i \in\{1, \ldots, k\}$, there exists $V_{i} \in \gamma$ such that $x_{i} \in V_{i} \subseteq U_{x_{i}}$. Then, $A \in\left\langle V_{1}, \ldots, V_{k}\right\rangle_{n} \subseteq \mathcal{A}$, with $\left\langle V_{1}, \ldots V_{k}\right\rangle_{n} \in \gamma^{\prime}$. Therefore:

$$
\gamma^{\prime}=\left\{\left\langle V_{1}, \ldots, V_{l}\right\rangle_{n} \mid V_{i} \in \beta \text { for each } i \in\{1, \ldots, l\} \text { and } l \in \mathbb{N}\right\}
$$

is a base of $\mathcal{F}_{n}(X)$. 
Suppose that $\mathcal{F}_{n}(X)$ has a countable base $\mathfrak{V}$. Let $\mathcal{B}=\{\bigcup \mathcal{V} \mid \mathcal{V} \in \mathfrak{V}\}$. By Theorem 3.2, part (4), the elements of $\mathcal{B}$ are open subsets of $X$, clearly, $\mathcal{B}$ is a countable set. We prove that $\mathcal{B}$ is a base for $X$. Let $U$ be an open subset of $X$ and let $x \in U$. Then $\langle U\rangle_{n}$ is an open subset of $\mathcal{F}_{n}(X)$ and $\{x\} \in\langle U\rangle_{n}$. Since $\mathfrak{V}$ is a base of $\mathcal{F}_{n}(X)$, there exists $\mathcal{V} \in \mathfrak{V}$ such that $\{x\} \in \mathcal{V} \subset\langle U\rangle_{n}$. Then $x \in \bigcup \mathcal{V} \subset U$. Note that $\bigcup \mathcal{V} \in \mathcal{B}$. Therefore, $\mathcal{B}$ is a countable base for $X$.

THEOREM 3.8. Let $X$ be a topological space and let $n \in \mathbb{N}$. Then $X$ is $T_{1}$ if and only if $\mathcal{F}_{n}(X)$ is $T_{1}$.

Proof. Suppose that $X$ is $T_{1}$, we see that $\mathcal{F}_{n}(X)$ is $T_{1}$. Let $A$ and $B$ be distinct points of $\mathcal{F}_{n}(X)$. Then $A \nsubseteq B$ or $B \nsubseteq A$. Without loss of generality, we assume that $A \nsubseteq B$. Hence, there exists $a \in A$ such that $a \notin B$. Let $B=\left\{b_{1}, \ldots, b_{l}\right\}$. Thus, for any $j \in\{1, \ldots, l\}, a \neq b_{j}$. Since $X$ is a $T_{1}$ space, for each $j \in\{1, \ldots, l\}$, there exist open subsets $U_{j}$ and $V_{j}$ of $X$ such that $a \in U_{j} \backslash V_{j}$ and $b_{j} \in V_{j} \backslash U_{j}$. Let $U=\bigcap_{i=1}^{l} U_{i}$. Note that, $U$ is an open subset of $X$ such that $A \in\langle X, U\rangle_{n} \backslash\left\langle V_{1}, \ldots, V_{l}\right\rangle_{n}$ and $B \in\left\langle V_{1}, \ldots, V_{l}\right\rangle_{n} \backslash\langle X, U\rangle_{n}$. Therefore, $\mathcal{F}_{n}(X)$ is a $T_{1}$ space.

Now, suppose that $\mathcal{F}_{n}(X)$ is $T_{1}$, we prove that $X$ is $T_{1}$. Let $x$ and $y$ be distinct points of $X$. Then $\{x\}$ and $\{y\}$ are different points of $\mathcal{F}_{n}(X)$. Since $\mathcal{F}_{n}(X)$ is a $T_{1}$ space, there exist open subsets $\mathcal{V}$ and $\mathcal{V}$ of $\mathcal{F}_{n}(X)$ such that $\{x\} \in \mathcal{V} \backslash \mathcal{V}$ and $\{y\} \in \mathcal{V} \backslash \mathcal{V}$. Then there exist open subsets $V_{1}, \ldots, V_{l}, U_{1}, \ldots, U_{r}$ of $X$ such that $\{x\} \in\left\langle U_{1}, \ldots, U_{r}\right\rangle_{n} \backslash\left\langle V_{1}, \ldots, V_{l}\right\rangle_{n}$ and $\{y\} \in\left\langle V_{1}, \ldots, V_{l}\right\rangle_{n} \backslash\left\langle U_{1}, \ldots, U_{r}\right\rangle_{n}$. This implies that $x \in \bigcap_{j=1}^{r} U_{j}$ and there exists $k \in\{1, \ldots, l\}$ such that $x \notin V_{k}$. Also, $y \in \bigcap_{j=1}^{l} V_{j}$ and there exists $m \in\{1, \ldots, r\}$ such that $y \notin U_{m}$. Let $U=\bigcap_{j=1}^{r} U_{j}$ and $V=\bigcap_{j=1}^{l} V_{j}$. Then $U$ and $V$ are open subsets of $X, x \in U \backslash V$ and $y \in V \backslash U$. Therefore, $X$ is a $T_{1}$ space.

THEOREM 3.9. Let $X$ be a topological space and let $n \in \mathbb{N}$. Then $X$ is Hausdorff if and only if $\mathcal{F}_{n}(X)$ is Hausdorff.

Proof. Suppose that $\mathcal{F}_{n}(X)$ is Hausdorff, we see that $X$ is Hausdorff. Let $x_{1}$ and $x_{2}$ be distinct points of $X$. Then $\left\{x_{1}\right\}$ and $\left\{x_{2}\right\}$ are distinct points of $\mathcal{F}_{n}(X)$. Since $\mathcal{F}_{n}(X)$ is Hausdorff, there exist open subsets $\mathcal{V}$ and $\mathcal{V}$ of $\mathcal{F}_{n}(X)$ such that $\left\{x_{1}\right\} \in \mathcal{V},\left\{x_{2}\right\} \in \mathcal{V}$ and $\mathcal{V} \cap \mathcal{V}=\varnothing$. By Theorem 3.1, there exist nonempty open subsets $U_{1}, \ldots, U_{n}, V_{1}, \ldots, V_{n}$ of $X$ such that $\left\{x_{1}\right\} \in\left\langle U_{1}, \ldots, U_{n}\right\rangle_{n} \subseteq \mathcal{V}$ and $\left\{x_{2}\right\} \in\left\langle V_{1}, \ldots, V_{n}\right\rangle_{n} \subseteq \mathcal{V}$. Note that $\left\langle U_{1}, \ldots, U_{n}\right\rangle_{n} \cap\left\langle V_{1} \ldots, V_{n}\right\rangle_{n}=\varnothing$. Let $U=\bigcap_{i=1}^{m} U_{i}$ and $V=\bigcap_{i=1}^{n} V_{i}$. Then $x_{1} \in U, x_{2} \in V$ and $U \cap V=\varnothing$. Therefore, $X$ is Hausdorff.

Now, suppose that $X$ is Hausdorff, we prove that $\mathcal{F}_{n}(X)$ is Hausdorff. Let $A$ and $B$ be distinct points of $\mathcal{F}_{n}(X)$. Then $A \nsubseteq B$ or $B \nsubseteq A$. Without loss of generality, we assume that $A \nsubseteq B$. Let $a \in A \backslash B$. Suppose that $B=\left\{b_{1}, \ldots, b_{m}\right\}$. Hence, for each $i \in\{1, \ldots, m\}, a \neq b_{i}$. Since $X$ is Hausdorff, for each $i \in\{1, \ldots, m\}$, there exist open subsets $U_{i}$ and $V_{i}$ of $X$ such that $a \in U_{i}, b_{i} \in V_{i}$ and $U_{i} \cap V_{i}=\varnothing$. Let $\mathcal{V}=\left\langle X, \bigcap_{i=1}^{m} U_{i}\right\rangle_{n}$ and $\mathcal{V}=\left\langle V_{1}, \ldots, V_{m}\right\rangle_{n}$. Then $\mathcal{V}$ and $\mathcal{V}$ are open subsets of $\mathcal{F}_{n}(X)$ such that $A \in \mathcal{V}$ and $B \in \mathcal{V}$. Now, we show that $\mathcal{V} \cap \mathcal{V}=\varnothing$. Suppose that $D \in \mathcal{V} \cap \mathcal{V}$. This implies that $D \cap\left(\bigcap_{i=1}^{m} U_{i}\right) \neq \varnothing$ and $D \subseteq \bigcup_{i=1}^{m} V_{i}$. Let $z \in D \cup\left(\bigcap_{i=1}^{m} U_{i}\right)$. Thus, $z \in \bigcup_{i=1}^{m} V_{i}$. Hence, there exists $i_{0} \in\{1, \ldots, m\}$ such that $z \in V_{i_{0}}$. Since $z \in \bigcap_{j=1}^{m} U_{i}$, we obtain that $z \in U_{i_{0}}$. Thus, $z \in V_{i_{0}} \cap U_{i_{0}}$, which is a contradiction. Therefore, $\mathcal{V} \cap \mathcal{V}=\varnothing$ and $\mathcal{F}_{n}(X)$ is a Hausdorff topological space.

THEOREM 3.10. Let $X$ be a topological space and let $n \in \mathbb{N}$. Then $X$ is pseudo-regular if and only if $\mathcal{F}_{n}(X)$ is pseudo-regular.

Proof. Suppose that $X$ is pseudo-regular. Let $\mathcal{V}$ be a nonempty open subset of $\mathcal{F}_{n}(X)$. Then, by Theorem 3.1, there exist nonempty open subsets $U_{1}, \ldots, U_{n}$ of $X$ such that $\left\langle U_{1}, \ldots, U_{n}\right\rangle_{n} \subseteq \mathcal{V}$. Since $X$ is pseudo-regular, for each $i \in\{1, \ldots, n\}$, there exists a nonempty open subset $V_{i}$ of $U_{i}$ such that $\operatorname{cl}_{X}\left(V_{i}\right) \subseteq U_{i}$. Note that $\left\langle V_{1}, \ldots, V_{n}\right\rangle_{n}$ is an open subset of $\mathcal{F}_{n}(X)$. We see that $\operatorname{cl}_{\mathcal{F}_{n}(X)}\left(\left\langle V_{1}, \ldots, V_{n}\right\rangle_{n}\right) \subseteq$ $\mathcal{V}$. By Theorem 3.2, part (3), we obtain that $\mathrm{cl}_{\mathcal{F}_{n}(X)}\left(\left\langle V_{1}, \ldots, V_{n}\right\rangle_{n}\right)=\left\langle\mathrm{cl}_{X}\left(V_{1}\right), \ldots, \mathrm{cl}_{X}\left(V_{n}\right)\right\rangle_{n}$. Clearly, $\left\langle\mathrm{cl}_{X}\left(V_{1}\right), \ldots, \operatorname{cl}_{X}\left(V_{n}\right)\right\rangle_{n} \subset\left\langle U_{1}, \ldots, U_{n}\right\rangle_{n}$. In consequence, we have that $\operatorname{cl}_{\mathcal{F}_{n}(X)}\left(\left\langle V_{1}, \ldots, V_{n}\right\rangle_{n}\right) \subseteq \mathcal{V}$. Therefore, $\mathcal{F}_{n}(X)$ is pseudo-regular.

Now, suppose that $\mathcal{F}_{n}(X)$ is pseudo-regular, we see that $X$ is pseudo-regular. Let $U$ be a nonempty open subset of $X$. Then, $\langle U\rangle_{n}$ is a nonempty open subset of $\mathcal{F}_{n}(X)$. Since $\mathcal{F}_{n}(X)$ is pseudo-regular, there exists a nonempty open subset $\mathcal{V}$ of $\mathcal{F}_{n}(X)$ such that $\operatorname{cl}_{\mathcal{F}_{n}(X)}(\mathcal{V}) \subseteq\langle U\rangle_{n}$. By Theorem 3.1, there exist nonempty open subsets $V_{1}, \ldots, V_{n}$ of $X$ such that $\left\langle V_{1}, \ldots, V_{n}\right\rangle_{n} \subseteq \mathcal{V}$. Now, by Theorem 3.2, 
part (3), we have that $\left\langle\mathrm{cl}_{X}\left(V_{1}\right), \ldots, \mathrm{cl}_{X}\left(V_{n}\right)\right\rangle_{n} \subseteq\langle U\rangle_{n}$. Let $V=\bigcup_{i=1}^{n} V_{i}$. Then $V$ is a nonempty open subset of $X$. It remains to verify that $\operatorname{cl}_{X}(V) \subseteq U$. Let $x \in \operatorname{cl}_{X}(V)$. Thus, there exists $i_{0} \in\{1, \ldots, n\}$ such that $x \in \operatorname{cl}_{X}\left(V_{i_{0}}\right)$. Without loss of generality, we assume that $i_{0}=1$. Let $y_{i} \in \operatorname{cl}_{X}\left(V_{i}\right)$, for each $i \in\{2, \ldots, n\}$. Note that:

$$
\left\{x, y_{2}, \ldots, y_{n}\right\} \in\left\langle\operatorname{cl}_{X}\left(V_{1}\right), \ldots, \operatorname{cl}_{X}\left(V_{n}\right)\right\rangle_{n} .
$$

Then $\left\{x, y_{2}, \ldots, y_{n}\right\} \in\langle U\rangle_{n}$. Hence, $\left\{x, y_{2}, \ldots, y_{n}\right\} \subseteq U$. Thus, $x \in U$ and $\operatorname{cl}_{X}(V) \subseteq U$. Therefore, $X$ is pseudo-regular.

THEOREM 3.11. Let $X$ be a topological space, let $U$ be an open subset of $X$ and let $n \in \mathbb{N}$. Then $\operatorname{cl}_{X}(U)$ is pseudo-regular if and only if $\left\langle\operatorname{cl}_{X}(U)\right\rangle_{n}$ is pseudo-regular.

Proof. Suppose that $\operatorname{cl}_{X}(U)$ is pseudo-regular. We see that $\left\langle\mathrm{cl}_{X}(U)\right\rangle_{n}$ is pseudo-regular. By Theorem 3.2, part (3), this is equivalent to verify that $\operatorname{cl}_{\mathcal{F}_{n}(X)}\left(\langle U\rangle_{n}\right)$ is pseudo-regular in $\mathcal{F}_{n}(X)$. Let $\mathcal{W}$ be a nonempty open subset of $\operatorname{cl}_{\mathcal{F}_{n}(X)}\left(\langle U\rangle_{n}\right)$. Then, $\mathcal{W}=\operatorname{cl}_{\mathcal{F}_{n}(X)}\left(\langle U\rangle_{n}\right) \cap \mathcal{W}^{\prime}$, with $\mathcal{W}^{\prime}$ an open subset of $\mathcal{F}_{n}(X)$. Since $\mathcal{W} \neq \varnothing$, we can take a point $B \in \mathcal{W}$. Thus, there exist open subsets $W_{1}, \ldots, W_{m}$ of $X$ such that $B \in\left\langle W_{1}, \ldots, W_{m}\right\rangle_{n} \cap \mathrm{cl}_{\mathcal{F}_{n}(X)}\left(\langle U\rangle_{n}\right) \subseteq \mathcal{W}$. By Theorem 3.2, part (3), we have that $\left\langle W_{1}, \ldots, W_{m}\right\rangle_{n} \cap \operatorname{cl}_{\mathcal{F}_{n}(X)}\left(\langle U\rangle_{n}\right)=\left\langle W_{1}, \ldots, W_{m}\right\rangle_{n} \cap\left\langle\operatorname{cl}_{X}(U)\right\rangle_{n}$. By [24, p. 8], we have that $\left\langle W_{1}, \ldots, W_{m}\right\rangle_{n} \cap\left\langle\operatorname{cl}_{X}(U)\right\rangle_{n}$ is equal to:

$$
\left\langle\bigcup_{i=1}^{n} W_{i} \cap \mathrm{cl}_{X}(U), \mathrm{cl}_{X}(U) \cap W_{1}, \ldots, \operatorname{cl}_{X}(U) \cap W_{m}\right\rangle_{n} .
$$

Hence,

$$
B \in\left\langle\bigcup_{i=1}^{n} W_{i} \cap \mathrm{cl}_{X}(U), \mathrm{cl}_{X}(U) \cap W_{1}, \ldots, \operatorname{cl}_{X}(U) \cap W_{m}\right\rangle_{n} .
$$

Thus, for each $i \in\{1, \ldots, m\}, B \cap\left(\operatorname{cl}_{X}(U) \cap W_{i}\right) \neq \varnothing$. For all $i \in\{1, \ldots, m\}$, let $T_{i}=W_{i} \cap \operatorname{cl}_{X}(U)$ and note that $T_{i} \neq \varnothing$. Since, for each $i \in\{1, \ldots, m\}, T_{i}$ is an open subset of $\operatorname{cl}_{X}(U)$ and $\operatorname{cl}_{X}(U)$ is pseudo-regular, for each $i \in\{1, \ldots, m\}$, there exists a nonempty open subset $T_{i}^{\prime}$ in $\operatorname{cl}_{X}(U)$ such that $\operatorname{cl}_{X}\left(T_{i}^{\prime}\right) \subseteq T_{i}$. Let $\mathcal{T}=\left\langle T_{1}^{\prime}, \ldots, T_{m}^{\prime}\right\rangle_{n}$. Then $\mathcal{T}$ is an open subset of $\left\langle\operatorname{cl}_{X}(U)\right\rangle_{n}$. Also, by Theorem 3.2, part (3), $\left\langle\operatorname{cl}_{X}(U)\right\rangle_{n}=\operatorname{cl}_{\mathcal{F}_{n}(X)}\left(\langle U\rangle_{n}\right)$. Thus, $\mathcal{T}$ is an open subset of $\operatorname{cl}_{\mathcal{F}_{n}(X)}\left(\langle U\rangle_{n}\right)$.

Observe that $\operatorname{cl}_{\mathcal{F}_{n}(X)}(\mathcal{T})=\left\langle\operatorname{cl}_{X}\left(T_{1}^{\prime}\right), \ldots, \operatorname{cl}_{X}\left(T_{m}^{\prime}\right)\right\rangle_{n}$. Then, since $\left\langle\operatorname{cl}_{X}\left(T_{1}^{\prime}\right), \ldots, \operatorname{cl}_{X}\left(T_{m}^{\prime}\right)\right\rangle_{n} \subseteq\left\langle W_{1} \cap\right.$ $\left.\operatorname{cl}_{X}(U), \ldots, W_{m} \cap \operatorname{cl}_{X}(U)\right\rangle_{n}, \operatorname{cl}_{\mathcal{F}_{n}(X)}(\mathcal{T}) \subseteq\left\langle W_{1} \cap \operatorname{cl}_{X}(U), \ldots, W_{m} \cap \operatorname{cl}_{X}(U)\right\rangle_{n}$. Finally, since $\left\langle W_{1} \cap\right.$ $\left.\operatorname{cl}_{X}(U), \ldots, W_{m} \cap \operatorname{cl}_{X}(U)\right\rangle_{n} \subseteq\left\langle W_{1}, \ldots, W_{m}\right\rangle_{n} \cap \operatorname{cl}_{\mathcal{F}_{n}(X)}\left(\langle U\rangle_{n}\right)$ and $\left\langle W_{1}, \ldots, W_{m}\right\rangle_{n} \cap \operatorname{cl}_{\mathcal{F}_{n}(X)}\left(\langle U\rangle_{n}\right) \subseteq \mathcal{W}$, we have that $\operatorname{cl}_{\mathcal{F}_{n}(X)}(\mathcal{T}) \subseteq \mathcal{W}$. Since $\mathcal{W}$ is arbitrary, we have that for each open subset $\mathcal{W}$ of $\operatorname{cl}_{\mathcal{F}_{n}(X)}\left(\langle U\rangle_{n}\right)$, there exists an open subset $\mathcal{T}$ of $\operatorname{cl}_{\mathcal{F}_{n}(X)}\left(\langle U\rangle_{n}\right)$ such that $\operatorname{cl}_{\mathcal{F}_{n}(X)}(\mathcal{T}) \subseteq \mathcal{W}$. Therefore, $\mathrm{cl}_{\mathcal{F}_{n}(X)}\left(\langle U\rangle_{n}\right)$ is pseudo-regular in $\mathcal{F}_{n}(X)$.

Now assume that $\left\langle\mathrm{cl}_{X}(U)\right\rangle_{n}$ is pseudo-regular, we prove that $\operatorname{cl}_{X}(U)$ is pseudo-regular. Let $V$ be a nonempty open subset of $\operatorname{cl}_{X}(U)$. We see that there exists a nonempty open subset $W$ of $V$ such that $\operatorname{cl}_{\operatorname{cl}_{X}(U)}(W) \subseteq V$. Note that $V=T \cap \operatorname{cl}_{X}(U)$, with $T$ nonempty open subset of $X$. Thus, $\langle V\rangle_{n}=\langle T\rangle_{n} \cap\left\langle\operatorname{cl}_{X}(U)\right\rangle_{n}$ is an open subset of $\left\langle\operatorname{cl}_{X}(U)\right\rangle_{n}$. Since $\left\langle\operatorname{cl}_{X}(U)\right\rangle_{n}$ is pseudoregular, we have that there exists a nonempty open subset $\mathcal{W}$ of $\langle V\rangle_{n}$ such that $\operatorname{cl}_{\left\langle\mathrm{cl}_{X}(U)\right\rangle_{n}}(\mathcal{W}) \subseteq$ $\langle V\rangle_{n}$. Observe that $\mathcal{W}=\mathcal{A} \cap\langle V\rangle_{n}$, with $\mathcal{A}$ open subset of $\mathcal{F}_{n}(X)$. It follows that, by Theorem 3.1, there exist nonempty open subsets $A_{1}, \ldots, A_{n}$ of $X$ such that $\left\langle A_{1}, \ldots, A_{n}\right\rangle_{n} \subseteq \mathcal{A}$. This implies that, $\left\langle A_{1}, \ldots, A_{n}\right\rangle_{n} \cap\langle V\rangle_{n} \subseteq \mathcal{A} \cap\langle V\rangle_{n}=\mathcal{W}$. Also note that [24, p. 8], $\operatorname{cl}_{\mathcal{F}_{n}(X)}\left(\left\langle A_{1}, \ldots, A_{n}\right\rangle_{n} \cap\langle V\rangle_{n}\right)=$ $\operatorname{cl}_{\mathcal{F}_{n}(X)}\left(\left\langle\bigcup_{i=1}^{n} A_{i} \cap V, V \cap A_{1}, \ldots, V \cap A_{n}\right\rangle_{n}\right), \operatorname{cl}_{\mathcal{F}_{n}(X)}\left(\left\langle\bigcup_{i=1}^{n} A_{i} \cap V, V \cap A_{1}, \ldots, V \cap A_{n}\right\rangle_{n}\right)=\left\langle\operatorname{cl}_{X}\left(\bigcup_{i=1}^{n} A_{i} \cap\right.\right.$ $\left.V), \operatorname{cl}_{X}\left(V \cap A_{1}\right), \ldots, \operatorname{cl}_{X}\left(V \cap A_{n}\right)\right\rangle_{n}$ and $\left\langle\operatorname{cl}_{X}\left(\bigcup_{i=1}^{n} A_{i} \cap V\right), \operatorname{cl}_{X}\left(V \cap A_{1}\right), \ldots, \operatorname{cl}_{X}\left(V \cap A_{n}\right)\right\rangle_{n} \subseteq\langle V\rangle_{n}$.

Let $W=\bigcup_{i=1}^{n}\left(V \cap A_{i}\right)$. We prove that $\operatorname{cl}_{\operatorname{cl}_{X}(U)}(W) \subseteq V$. Recall that $\operatorname{cl}_{\operatorname{cl}_{X}(U)}(W)=\operatorname{cl}_{X}(W) \cap \operatorname{cl}_{X}(U)$. Since $\operatorname{cl}_{X}(W) \subseteq \operatorname{cl}_{X}(U)$, we have that $\operatorname{cl}_{X}(W) \cap \operatorname{cl}_{X}(U)=\operatorname{cl}_{X}(W)$. Thus, we only need to prove that $\operatorname{cl}_{X}(W) \subseteq V$. Let $x \in \operatorname{cl}_{X}(W)=\operatorname{cl}_{X}\left(\bigcup_{i=1}^{n}\left(V \cap A_{i}\right)\right)=\bigcup_{i=1}^{n} \operatorname{cl}_{X}\left(V \cap A_{i}\right)$. Then, there exists $i_{0} \in\{1, \ldots, n\}$ such that $x \in \operatorname{cl}_{X}\left(V \cap A_{i_{0}}\right)$. Without loss of generality, we assume that $i_{0}=1$. For each $i \in\{2, \ldots, n\}$, 
let $y_{i} \in \operatorname{cl}_{X}\left(V \cap A_{i}\right)$. Then:

$$
\left\{x, y_{2}, \ldots, y_{n}\right\} \in\left\langle\operatorname{cl}_{X}\left(V \cap A_{1}\right), \ldots, \operatorname{cl}_{X}\left(V \cap A_{n}\right)\right\rangle_{n} .
$$

Thus, $\left\{x, y_{2}, \ldots, y_{n}\right\} \subseteq V$. In particular, $x \in V$. Since $x \in \operatorname{cl}_{X}(W)$ is arbitrary, we conclude that $\operatorname{cl}_{X}(W) \subseteq V$. Therefore, $\operatorname{cl}_{X}(U)$ is pseudo-regular.

THEOREM 3.12. Let $X$ be a topological space, let $U$ be an open subset of $X$ and let $n \in \mathbb{N}$. If $\operatorname{cl}_{X}(U)$ is compact in $X$, then $\mathrm{cl}_{\mathcal{F}_{n}(X)}\left(\langle U\rangle_{n}\right)$ is compact in $\mathcal{F}_{n}(X)$.

Proof. Suppose that $\mathrm{cl}_{X}(U)$ is compact. It follows that $\left(\mathrm{cl}_{X}(U)\right)^{n}$ is compact. By Theorem 3.2, part (1), $f_{n}$ is a map. Hence, $f_{n}\left(\left(\mathrm{cl}_{X}(U)\right)^{n}\right)$ is compact. Moreover, by Theorem 3.2, part (2), we have that $f_{n}\left(\operatorname{cl}_{X}(U)^{n}\right)=\mathcal{F}_{n}\left(\operatorname{cl}_{X}(U)\right)$. Thus, $\mathcal{F}_{n}\left(\operatorname{cl}_{X}(U)\right)$ is compact. By Theorem 3.2, part $(3), \operatorname{cl}_{\mathcal{F}_{n}(X)}\left(\langle U\rangle_{n}\right)=$ $\left\langle\mathrm{cl}_{X}(U)\right\rangle_{n}$. On the other hand, $\mathcal{F}_{n}\left(\operatorname{cl}_{X}(U)\right)=\left\langle\mathrm{cl}_{X}(U)\right\rangle_{n}$. Therefore, $\mathrm{cl}_{\mathcal{F}_{n}(X)}\left(\langle U\rangle_{n}\right)$ is compact.

As a consequence of Theorems 3.11 and 3.12, we have the following:

THEOREM 3.13. Let $X$ be a topological space and let $n \in \mathbb{N}$. If $X$ is partially compact and pseudo-regular, then $\mathcal{F}_{n}(X)$ is partially compact and pseudo-regular.

The following result is a consequence of the proof of [17, Theorem 3.10].

THEOREM 3.14. Let $X$ be a topological space and let $n \in \mathbb{N}$. If $\mathcal{D}$ is a dense subset of $\mathcal{F}_{n}(X)$, then $\bigcup \mathcal{D}$ is a dense subset of $X$.

As a consequence of Theorem 3.14 and the proof of [17, Theorem 3.10], we have:

THEOREM 3.15. Let $X$ be a topological space and let $A \subseteq X$. Then, $\mathcal{F}_{n}(A)$ is dense in $\mathcal{F}_{n}(X)$ if and only if $A$ is dense in $X$.

THEOREM 3.16. Let $X$ be a topological space and let $n \in \mathbb{N}$. Then $X$ is perfect if and only if $\mathcal{F}_{n}(X)$ is perfect.

Proof. Suppose that $\mathcal{F}_{n}(X)$ is perfect, we see that $X$ is perfect. Suppose that $X$ has at least one isolated point, say $x_{0}$. Then $\left\{x_{0}\right\}$, is an open subset of $X$. Hence, $\left\langle\left\{x_{0}\right\}\right\rangle_{n}=\left\{\left\{x_{0}\right\}\right\}$ is an open subset of $\mathcal{F}_{n}(X)$. Thus, $\mathcal{F}_{n}(X)$ has an isolated point.

Now, suppose that $\mathcal{F}_{n}(X)$ has at least one isolated point, say $A$. Then $\{A\}$ is an open subset of $\mathcal{F}_{n}(X)$. By Theorem 3.1, there exists nonempty open subsets $V_{1}, \ldots, V_{n}$ of $X$ such that $A \in$ $\left\langle V_{1}, \ldots, V_{n}\right\rangle_{n} \subset\{A\}$. Hence, $A \subset \bigcup_{i=1}^{n} V_{i} \subset \bigcup\{A\}=A$. Since $A$ is a finite subset of $X$, this implies that $X$ has an isolated point.

THEOREM 3.17. Let $X$ be a topological space and let $n \in \mathbb{N}$. If $\mathcal{F}_{n}(X)$ has no quasi-isolated points, then $X$ has no quasi-isolated points.

Proof. Suppose that $X$ has at least one quasi-isolated point, say $a$. Then, there exists a dense subset $A$ of $X$ such that $a \in A$ and $a$ is an isolated point of $A$. By Theorem 3.15, $\mathcal{F}_{n}(A)$ is a dense subset of $\mathcal{F}_{n}(X)$. Note that $\{a\} \in \mathcal{F}_{n}(A)$. Now, we see that $\{a\}$ is an isolated point of $\mathcal{F}_{n}(A)$. Since $a$ is an isolated point of $A$, there exists an open subset $U$ of $X$ such that $U \cap A=\{a\}$. Hence, $\langle U\rangle_{n}$ is an open subset of $\mathcal{F}_{n}(X)$ such that $\{a\} \in\langle U\rangle_{n}$. Suppose that there exists $B \in\left[\langle U\rangle_{n} \cap \mathcal{F}_{n}(A)\right] \backslash\{\{a\}\}$. Then $B \subset U \cap A$ and $B \neq\{a\}$. Let $b \in B \backslash\{a\}$. Hence, $b \in(U \cap A) \backslash\{a\}$, which is a contradiction. Thus, $\langle U\rangle_{n} \cap \mathcal{F}_{n}(A)=\{\{a\}\}$. Therefore, $\{a\}$ is an isolated point of $\mathcal{F}_{n}(A)$ and $\{a\}$ is a quasi-isolated point of $\mathcal{F}_{n}(X)$.

THEOREM 3.18. Let $X$ be a topological space and let $n \in \mathbb{N}$. If $X$ has no quasi-isolated points, then $\mathcal{F}_{n}(X)$ is perfect.

Proof. Suppose $\mathcal{F}_{n}(X)$ is not perfect. Then, by Theorem 3.16, $X$ has an isolated point, say $x$. Let $A$ be any dense subset of $X$. Then $x \in A$ and, clearly, $\{x\}$ is an isolated point of $A$. Therefore, $x$ is a quasi-isolated point of $X$.

By Theorem 3.17 and [34, Lemma 2.5], we have the following result. 
THEOREM 3.19. Let $X$ be a $T_{1}$ topological space and let $n \in \mathbb{N}$. Then $X$ has no quasi-isolated points if and only if $\mathcal{F}_{n}(X)$ has no quasi-isolated points.

\section{TOPOLOGICAL TRANSITIVITY ON SYMMETRIC PRODUCTS}

In this section we study the possible transitivity of the induced function on the symmetric products. We begin with the following:

THEOREM 4.1. Let $X$ be a topological space, let $f: X \rightarrow X$ be a function and let $n \geq 2$. If there exist nonempty open subsets $U$ and $V$ of $X$ such that $U \cap V=\varnothing$ and for each $k \in \mathbb{N}, f^{-k}(U) \subset V$, then $\mathcal{F}_{n}(f): \mathcal{F}_{n}(X) \rightarrow \mathcal{F}_{n}(X)$ is not $\mathbb{Z}$-transitive.

Proof. Let $U$ and $V$ be nonempty open subsets of $X$ such that $U \cap V=\varnothing$ and for each $k \in \mathbb{N}$, $f^{-k}(U) \subset V$. Let $W$ be a nonempty open subset of $U$. Then $\langle W\rangle_{n}$ and $\langle U\rangle_{n}$ are nonempty open subsets of $\mathcal{F}_{n}(X)$. Suppose that $\mathcal{F}_{n}(f): \mathcal{F}_{n}(X) \rightarrow \mathcal{F}_{n}(X)$ is $\mathbb{Z}$-transitive. Hence, we have that $N_{\mathcal{F}_{n}(f)}\left(\langle W\rangle_{n},\langle U\rangle_{n}\right) \neq \varnothing$. Thus, there exists $k \in \mathbb{Z}$ such that $\left[\mathcal{F}_{n}(f)\right]^{k}\left(\langle W\rangle_{n}\right) \cap\langle U\rangle_{n} \neq \varnothing$. Now, let $A \in\langle W\rangle_{n}$ be such that $\left[\mathcal{F}_{n}(f)\right]^{k}(A) \in\langle U\rangle_{n}$. Then $A \subset W$ and $f^{k}(A) \subset U$. Thus, we have the following cases:

(a) $k>0$. In this case, we have that $A \subset U$ and $A \subset f^{-k}(U)$. Since $f^{-k}(U) \subset V$, we obtain that $A \subset U \cap V$. In consequence, $U \cap V \neq \varnothing$, which is a contradiction.

(b) $k=0$. For this case, $U \subseteq V$. Thus, $U \cap V \neq \varnothing$, which is a contradiction.

(c) $k<0$. In this case, we have that $-k>0, f^{k}(A) \subset f^{k}(U)$ and $f^{k}(A) \subset U$. Since $f^{k}(U) \subset V$, we have that $f^{k}(A) \subset U \cap V$, then $U \cap V \neq \varnothing$, which is a contradiction.

Therefore, $\mathcal{F}_{n}(f): \mathcal{F}_{n}(X) \rightarrow \mathcal{F}_{n}(X)$ is not $\mathbb{Z}$-transitive.

THEOREM 4.2. Let $(X, d)$ be a metric space, let $f: X \rightarrow X$ be a map, let $n \geq 2$ and let $L \in \mathbb{R}$ be such that $0<L \leq 1$. If there exists $a \in X$ such that $f(a) \neq a$ and for each $x \in X$ and every $k \in \mathbb{N}$, $d(x, a) \leq L \cdot d\left(f^{k}(x), f(a)\right)$, then $\mathcal{F}_{n}(f): \mathcal{F}_{n}(X) \rightarrow \mathcal{F}_{n}(X)$ is not $\mathbb{Z}$-transitive.

Proof. Let $a \in X$ be such that $f(a) \neq a$ and suppose that for each $x \in X$, we have that $d(x, a) \leq$ $L \cdot d\left(f^{k}(x), f(a)\right)$. Let $\varepsilon=\frac{d(a, f(a))}{2}$. Thus, $\varepsilon>0$. We define the subsets of $X, U=B(f(a), \varepsilon)=\{x \in X \mid$ $d(f(a), x)<\varepsilon\}$ and $V=B(a, L \cdot \varepsilon)$. Then $U$ and $V$ are nonempty open subsets of $X$.

We prove that $U \cap V=\varnothing$. Suppose that $U \cap V \neq \varnothing$. Let $b \in U \cap V$. Then $d(b, f(a))<\varepsilon$ and $d(a, b)<L \cdot \varepsilon$. By the triangle inequality, we have that $d(a, f(a)) \leq d(a, b)+d(b, f(a))<L \cdot \varepsilon+\varepsilon$. Since $L \leq 1, L \cdot \varepsilon \leq \varepsilon$. Hence, $d(a, f(a))<2 \varepsilon$. Thus, $d(a, f(a))<d(a, f(a))$, which is a contradiction. Therefore, $U \cap V=\varnothing$.

Now, we show that, for each $k \in \mathbb{N}, f^{-k}(U) \subseteq V$. Let $k \in \mathbb{N}$ and let $x \in f^{-k}(U)$. Then $f^{k}(x) \in U$. Thus, $d\left(f^{k}(x), f(a)\right)<\varepsilon$. Thus, $L \cdot d\left(f^{k}(x), f(a)\right)<L \cdot \varepsilon$. Since $d(x, a) \leq L \cdot d\left(f^{k}(x), f(a)\right)$, we have that $d(x, a)<L \cdot \varepsilon$. Hence, $x \in V$. Therefore, $f^{-k}(U) \subseteq V$.

By Theorem 4.1, we obtain that $\mathcal{F}_{n}(f): \mathcal{F}_{n}(X) \rightarrow \mathcal{F}_{n}(X)$ is not $\mathbb{Z}$-transitive.

THEOREM 4.3. Let $X$ be a topological space, let $f: X \rightarrow X$ be a function and let $n \geq 2$. If there exist nonempty disjoint open subsets $U$ and $V$ of $X$ and for each $k \in \mathbb{N}, f^{k}(V) \subset U$, then $\mathcal{F}_{n}(f): \mathcal{F}_{n}(X) \rightarrow$ $\mathcal{F}_{n}(X)$ is not $\mathbb{Z}$-transitive.

Proof. Suppose that there exist nonempty disjoint open subsets $U$ and $V$ of $X$ and for each $k \in \mathbb{N}$, $f^{k}(V) \subset U$. Let $W$ be a nonempty open subset of $X$ such that $W \subset V$. Then $\langle W\rangle_{n}$ and $\langle V\rangle_{n}$ are nonempty open subsets of $\mathcal{F}_{n}(X)$. Suppose that $\mathcal{F}_{n}(f): \mathcal{F}_{n}(X) \rightarrow \mathcal{F}_{n}(X)$ is $\mathbb{Z}$-transitive. Hence, $N_{\mathcal{F}_{n}(f)}\left(\langle W\rangle_{n},\langle U\rangle_{n}\right) \neq \varnothing$. Thus, there exists $k \in \mathbb{Z}$ such that $\left[\mathcal{F}_{n}(f)\right]^{k}\left(\langle W\rangle_{n}\right) \cap\langle U\rangle_{n} \neq \varnothing$. Let $A \in\langle W\rangle_{n}$ be such that $\left[\mathcal{F}_{n}(f)\right]^{k}(A) \in\langle V\rangle_{n}$. This implies that $A \subset W$ and $f^{k}(A) \subset V$. Then we have the following cases:

(a) $k>0$. In this case, $f^{k}(A) \subset f^{k}(W) \subset f^{k}(V)$ and $f^{k}(A) \subset V$. Since $f^{k}(V) \subset U, f^{k}(A) \subset V \cap U$. Consequently, $U \cap V \neq \varnothing$, a contradiction.

(b) $k=0$. For this case, $U \subseteq V$. Thus, $U \cap V \neq \varnothing$, a contradiction. 
(c) $k<0$. In this case, we have that $-k>0$. Also, $A \subset W \subset V$ and $A \subset f^{-k}(V)$. Since $f^{-k}(V) \subset U$, we obtain that $A \subset U \cap V$. Thus, $U \cap V \neq \varnothing$, a contradiction.

Therefore, $\mathcal{F}_{n}(f): \mathcal{F}_{n}(X) \rightarrow \mathcal{F}_{n}(X)$ is not $\mathbb{Z}$-transitive.

THEOREM 4.4. Let $(X, d)$ be a metric space, let $f: X \rightarrow X$ be a function, let $n \geq 2$ and let $L \in \mathbb{R}$ be such that $L \geq 1$. If there exists $a \in X$ such that $f(a) \neq a$ and for each $x \in X \backslash\{a\}$ and every $k \in \mathbb{N}$, $L \cdot d\left(f^{k}(x), f(a)\right) \leq d(x, a)$, then $\mathcal{F}_{n}(f): \mathcal{F}_{n}(X) \rightarrow \mathcal{F}_{n}(X)$ is not $\mathbb{Z}$-transitive.

Proof. Let $a \in X$ be such that $f(a) \neq a$ and suppose that for each $x \in X$ and for each $k \in \mathbb{N}$, $L \cdot d\left(f^{k}(x), f(a)\right) \leq d(x, a)$. Let $\varepsilon=\frac{d(a, f(a))}{2}$. Then $\varepsilon>0$. Let $U=B\left(f(a), \frac{\varepsilon}{L}\right)$ and let $V=B(a, \varepsilon)$. Hence, $U$ and $V$ are nonempty open subsets of $X$.

We prove that $U \cap V=\varnothing$. Suppose that $U \cap V \neq \varnothing$. Let $b \in U \cap V$. Then $d(b, f(a))<\frac{\varepsilon}{L}$ and $d(a, b)<\varepsilon$. By the triangle inequality, we obtain that $d(a, f(a)) \leq d(a, b)+d(b, f(a))<\varepsilon+\frac{\varepsilon}{L}$. Since $1 \leq L, \frac{\varepsilon}{L} \leq \varepsilon$. Hence, $d(a, f(a))<2 \varepsilon$. Thus, $d(a, f(a))<d(a, f(a))$, which is a contradiction. Therefore, $U \cap V=\varnothing$.

Now, we see that $f^{k}(V) \subseteq U$. Let $x \in V$. Then $d(x, a)<\varepsilon$. Since $L \cdot d\left(f^{k}(x), f(a)\right) \leq d(x, a)$, $L \cdot d\left(f^{k}(x), f(a)\right)<\varepsilon$. Hence, $d\left(f^{k}(x), f(a)\right)<\frac{\varepsilon}{L}$. Thus, $f^{k}(x) \in U$. Therefore, $f^{k}(V) \subseteq U$.

By Theorem 4.3, the function $\mathcal{F}_{n}(f): \mathcal{F}_{n}(X) \rightarrow \mathcal{F}_{n}(X)$ is not $\mathbb{Z}$-transitive.

THEOREM 4.5. Let $(X, d)$ be a metric space, let $f: X \rightarrow X$ be a surjective function and let $n \geq 2$. If $f$ is an isometry, then $\mathcal{F}_{n}(f): \mathcal{F}_{n}(X) \rightarrow \mathcal{F}_{n}(X)$ is not $\mathbb{Z}$-transitive.

Proof. Suppose that $f$ is an isometry and that $\mathcal{F}_{n}(f)$ is $\mathbb{Z}$-transitive. Let $x, y \in X$ be such that $x \neq y$ and $r=d(x, y)$. Then $r>0$. Let $U=B\left(x, \frac{r}{4}\right)$ and let $V=B\left(y, \frac{r}{4}\right)$. Note that $U \cap V=\varnothing$. Let $z_{1}, z_{2} \in U$ be such that $z_{1} \neq z_{2}$. Then there exist open subsets $U_{1}$ and $U_{2}$ in $X$ such that $U_{1} \cup U_{2} \subseteq U$ and $U_{1} \cap U_{2}=\varnothing$. Hence, $\left\langle U_{1}, V\right\rangle_{n}$ and $\left\langle U_{2}\right\rangle_{n}$ are nonempty open subsets of $\mathcal{F}_{n}(X)$. Since $\mathcal{F}_{n}(f)$ is $\mathbb{Z}$ transitive, there exists $k \in \mathbb{Z}$ such that $\left[\mathcal{F}_{n}(f)\right]^{k}\left(\left\langle U_{1}, V\right\rangle_{n}\right) \cap\left\langle U_{2}\right\rangle_{n} \neq \varnothing$. Let $A \in\left\langle U_{1}, V\right\rangle_{n}$ be such that $\left[\mathcal{F}_{n}(f)\right]^{k}(A) \in\left\langle U_{2}\right\rangle_{n}$. Then $A \cap U_{1} \neq \varnothing, A \cap V \neq \varnothing$ and $f^{k}(A) \subseteq U_{2}$. Let $a \in A \cap U_{1}$ and let $b \in A \cap V$. Observe that, $d(x, y) \leq d(x, a)+d(a, b)+d(b, y)$, this implies that, $d(x, y)<\frac{r}{4}+d(a, b)+\frac{r}{4}$. Thus, $r<\frac{r}{2}+d(a, b)$. Therefore, $\frac{r}{2}<d(a, b)$.

We consider the following cases:

(a) $k>0$. In this case, since $f^{k}(A) \subseteq U_{2}, f^{k}(a) \in U_{2}$ and $f^{k}(b) \in U_{2}$. Hence, $d\left(f^{k}(a), f^{k}(b)\right) \leq \frac{r}{2}$. This implies that $\frac{r}{2}<d(a, b)=d\left(f^{k}(a), f^{k}(b)\right) \leq \frac{r}{2}$. Thus, $\frac{r}{2}<\frac{r}{2}$, a contradiction.

(b) $k=0$. For this case, $f^{k}(A)=A$. Then $U \cap V \neq \varnothing$, a contradiction.

(c) $k<0$. In this case, $-k>0$. Then $f^{k}(A) \subseteq U_{2}$. Thus, we obtain that $f^{-k}\left(f^{k}(A)\right) \subseteq f^{-k}\left(U_{2}\right)$. Thus, $A \subseteq f^{-k}\left(U_{2}\right)$. Since $a, b \in A$, we have that $a, b \in f^{-k}\left(U_{2}\right)$. Hence, there exist elements $c, d \in U_{2}$ such that $f^{-k}(c)=a$ and $f^{-k}(d)=b$. Since $c, d \in U_{2}, d(c, d) \leq \frac{r}{2}$. However, $d(a, b)=$ $d\left(f^{-k}(c), f^{-k}(d)\right)=d(c, d)$. Thus, $\frac{r}{2}<\frac{r}{2}$, a contradiction.

From these three cases, we conclude that the function $\mathcal{F}_{n}(f): \mathcal{F}_{n}(X) \rightarrow \mathcal{F}_{n}(X)$ is not $\mathbb{Z}$-transitive.

By Theorem 4.5 and the diagram of Figure 1, we have the following result, compare with [7, Theorem 4.5].

THEOREM 4.6. Let $X$ be a metric space, let $f: X \rightarrow X$ be a surjective function and let $n \geq 2$. Let $\mathcal{M}$ be one of the following classes of functions: $\mathbb{Z}$-transitive, $\mathbb{Z}_{+}$-transitive, orbit-transitive, strictly orbit-transitive, $\omega$-transitive, transitive, $T T_{++}$, strongly transitive, chaotic, totally transitive, weakly mixing, mixing or exact. If $f$ is an isometry, then $\mathcal{F}_{n}(f) \notin \mathcal{M}$.

DEFINITION 4.7. Let $S^{1}=\left\{e^{2 \pi i \alpha}: \alpha \in[0,1]\right\}$ and let $\theta$ be an irrational number. We define the irrational rotation $R: S^{1} \rightarrow S^{1}$ given by $R\left(e^{2 \pi i \alpha}\right)=e^{2 \pi i \theta}\left(e^{2 \pi i \alpha}\right)=e^{2 \pi i(\theta+\alpha)}$, for each $\alpha \in[0,1]$ or simply;

$$
R(z)=\left(e^{2 \pi i \theta}\right) z, \text { for each } z \in S^{1} .
$$

Next we present some of the properties of the irrational rotation function, which are known in the literature, see [41, p. 261]. 
PROPOSITION 4.8. The irrational rotation function $R$ of Definition 4.7 is: an isometry, minimal, totally transitive, strongly transitive, transitive, irreducible and semi-open map.

The following result generalizes [21, Theorem 4.3] to arbitrary topological spaces and functions not necessarily continuous.

THEOREM 4.9. Let $X$ be a topological space, let $f: X \rightarrow X$ be a function and let $n \in \mathbb{N}$. Then $\mathcal{F}_{n}(f)$ is mixing if and only if $f$ is mixing.

Proof. Suppose that $\mathcal{F}_{n}(f)$ is mixing. We see that $f$ is mixing. Let $U$ and $V$ be open subsets of $X$. Then $\langle U\rangle_{n}$ and $\langle V\rangle_{n}$ are open subsets of $\mathcal{F}_{n}(X)$. Since $\mathcal{F}_{n}(f)$ is mixing, there exists $N \in \mathbb{N}$ such that $\left[\mathcal{F}_{n}(f)\right]^{k}\left(\langle U\rangle_{n}\right) \cap\langle V\rangle_{n} \neq \varnothing$, for each $k \geq N$. We show that for each $k \geq N, f^{k}(U) \cap V \neq \varnothing$. Let $k \geq N$. Since $\left[\mathcal{F}_{n}(f)\right]^{k}\left(\langle U\rangle_{n}\right) \cap\langle V\rangle_{n} \neq \varnothing$, there exists $B \in\langle U\rangle_{n}$ such that $\left[\mathcal{F}_{n}(f)\right]^{k}(B) \in\langle V\rangle_{n}$. Thus, $B \subset U$ and $f^{k}(B) \subseteq V$. Then $f^{k}(U) \cap V \neq \varnothing$. Therefore, $f$ is mixing.

Next, suppose that $f$ is mixing, we prove that $\mathcal{F}_{n}(f)$ is mixing. Let $\mathcal{V}$ and $\mathcal{V}$ be nonempty open subsets of $\mathcal{F}_{n}(X)$. Then, by Theorem 3.1, there exist nonempty open subsets $U_{1}, \ldots, U_{n}, V_{1}, \ldots, V_{n}$ of $X$ such that $\left\langle U_{1}, \ldots, U_{n}\right\rangle_{n} \subseteq \mathcal{V}$ and $\left\langle V_{1}, \ldots, V_{n}\right\rangle_{n} \subseteq \mathcal{V}$. Since $f$ is mixing, for each $i \in\{1, \ldots, n\}$, there exists $N_{i} \in \mathbb{N}$ such that $f^{k}\left(U_{i}\right) \cap V_{i} \neq \varnothing$, for every $k \geq N_{i}$. Let $N=\max \left\{N_{1}, \ldots, N_{n}\right\}$. Note that $f^{k}\left(U_{i}\right) \cap V_{i} \neq \varnothing$, for each $k \geq N$ and every $i \in\{1, \ldots, n\}$. Let $k \geq N$. Then for each $i \in\{1, \ldots, n\}$, $f^{k}\left(U_{i}\right) \cap V_{i} \neq \varnothing$. Hence, for every $i \in\{1, \ldots, n\}$, let $x_{i} \in U_{i}$ be such that $f^{k}\left(x_{i}\right) \in V_{i}$. Let $A=\left\{x_{1}, \ldots, x_{n}\right\}$. Observe that $A \in\left\langle U_{1}, \ldots, U_{n}\right\rangle_{n}$ and $\left[\mathcal{F}_{n}(f)\right]^{k}(A) \in\left\langle V_{1}, \ldots, V_{n}\right\rangle_{n}$. Thus, $\left[\mathcal{F}_{n}(f)\right]^{k}(\mathcal{V}) \cap \mathcal{V} \neq \varnothing$. Hence, for each $k \geq N,\left[\mathcal{F}_{n}(f)\right]^{k}(\mathcal{U}) \cap \mathcal{V} \neq \varnothing$. Therefore, $\mathcal{F}_{n}(f)$ is mixing.

The following theorem generalizes the corresponding part of [6, Theorem 10.1] to arbitrary topological spaces and functions not necessarily continuous.

THEOREM 4.10. Let $X$ be a topological space, let $f: X \rightarrow X$ be a function and let $n \in \mathbb{N}$. Then $\mathcal{F}_{n}(f)$ is semi-open if and only if $f$ is semi-open.

Proof. Suppose that $\mathcal{F}_{n}(f)$ is semi-open, we see that $f$ is semi-open. Let $U$ be a nonempty open subset of $X$. Then, $\langle U\rangle_{n}$ is a nonempty open subset of $\mathcal{F}_{n}(X)$. Since $\mathcal{F}_{n}(f)$ is semi-open, we obtain that $\operatorname{int}_{\mathcal{F}_{n}(X)}\left(\mathcal{F}_{n}(f)\left(\langle U\rangle_{n}\right)\right) \neq \varnothing$. Thus, there exists a nonempty open subset $\mathcal{V}$ of $\mathcal{F}_{n}(X)$ such that $\mathcal{V} \subseteq \mathcal{F}_{n}(f)\left(\langle U\rangle_{n}\right)$. Let $V=U \mathcal{V}$. By Theorem 3.2, part (4), we have that $V$ is an open subset of $X$. Now, we show that $V \subseteq f(U)$. Let $z \in V$. Then there exists $B \in \mathcal{V}$ such that $z \in B$. Hence, $B \in \mathcal{F}_{n}(f)\left(\langle U\rangle_{n}\right)$. Thus, there exists $A \in\langle U\rangle_{n}$ such that $\mathcal{F}_{n}(f)(A)=B$. This implies that $z \in f(A)$. Since $A \subseteq U$, $f(a)=z \in f(U)$. Hence, $V \subseteq f(U)$ and $\operatorname{int}_{X}(f(U)) \neq \varnothing$. Therefore, $f$ is semi-open.

Now, suppose that $f$ is semi-open, the proof that $\mathcal{F}_{n}(f)$ is semi-open is essentially the same as the proof of (1) implies (2) of [6, Theorem 10.1].

The following theorem also extends [16, Theorem 13] to arbitrary topological spaces and functions not necessarily continuous.

THEOREM 4.11. Let $X$ be a topological space, let $f: X \rightarrow X$ be a function and let $n \in \mathbb{N}$. Then $\mathcal{F}_{n}(f)$ is exact if and only if $f$ is exact.

Proof. Suppose that $f$ is exact, we show that $\mathcal{F}_{n}(f)$ is exact. Let $\mathcal{V}$ be a nonempty open subset of $\mathcal{F}_{n}(X)$. Then, by Theorem 3.1, there exist nonempty open subsets $U_{1}, \ldots, U_{n}$ of $X$ such that $\left\langle U_{1}, \ldots, U_{n}\right\rangle_{n} \subseteq \mathcal{V}$. Since $f$ is exact, there exist $k_{1}, \ldots, k_{n} \in \mathbb{N}$ such that $f^{k_{i}}\left(U_{i}\right)=X$. Let $k=\max \left\{k_{1}, \ldots, k_{n}\right\}$. Since $f$ is exact and by the diagram of Figure 1, we obtain that $f$ is surjective. Thus, $f^{k}\left(U_{i}\right)=X$, for each $i \in\{1, \ldots, n\}$. Now, we show that:

$$
\mathcal{F}_{n}(X) \subseteq\left[\mathcal{F}_{n}(f)\right]^{k}\left(\left\langle U_{1}, \ldots, U_{n}\right\rangle_{n}\right) .
$$

Let $B=\left\{b_{1}, \ldots, b_{r}\right\} \in \mathcal{F}_{n}(X)$, with $r \leq n$. Define $C=\left\{b_{1}, \ldots, b_{r}, b_{r+1}, \ldots, b_{n}\right\}$, where $b_{r}=$ $b_{r+1}=\cdots=b_{n}$. Then, for each $i \in\{1, \ldots, n\}, b_{i} \in X=f^{k}\left(U_{i}\right)$. Thus, for every $i \in\{1, \ldots, n\}$, there exists $a_{i} \in U_{i}$ such that $f^{k}\left(a_{i}\right)=b_{i}$. Let $A=\left\{a_{1}, \ldots, a_{n}\right\}$. Then $A \in\left\langle U_{1}, \ldots, U_{n}\right\rangle_{n}$ and $\left[\mathcal{F}_{n}(f)\right]^{k}(A)=C=B$. Thus, $B \in\left[\mathcal{F}_{n}(f)\right]^{k}\left(\left\langle U_{1}, \ldots, U_{n}\right\rangle_{n}\right)$. Hence, $\mathcal{F}_{n}(X)=\left[\mathcal{F}_{n}(f)\right]^{k}\left(\left\langle U_{1}, \ldots, U_{n}\right\rangle_{n}\right)$. Therefore, $\left[\mathcal{F}_{n}(f)\right]^{k}(\mathcal{U})=\mathcal{F}_{n}(X)$, and $f$ is exact. 
The proof of the converse implication is essentially the same as the one given in [16, Theorem 13].

The proof of the following two results generalize [21, Theorem 4.5] to arbitrary topological spaces and functions that are not necessarily continuous.

THEOREM 4.12. Let $X$ be a topological space, let $f: X \rightarrow X$ be a function and let $n \in \mathbb{N}$. If $\mathcal{F}_{n}(f)$ is transitive, then $f$ is transitive.

Proof. Suppose that $\mathcal{F}_{n}(f)$ is transitive, we see that $f$ is transitive. Let $U$ and $V$ be nonempty open subsets of $X$. Then $\langle U\rangle_{n}$ and $\langle V\rangle_{n}$ are nonempty open subsets of $\mathcal{F}_{n}(X)$. Since $\mathcal{F}_{n}(f)$ is transitive, there exists $k \in \mathbb{N}$ such that $\left[\mathcal{F}_{n}(f)\right]^{k}\left(\langle U\rangle_{n}\right) \cap\langle V\rangle_{n} \neq \varnothing$. Thus, there exists $A \in\langle U\rangle_{n}$ such that $\left[\mathcal{F}_{n}(f)\right]^{k}(A) \in\langle V\rangle_{n}$. This implies that, $f^{k}(U) \cap V \neq \varnothing$. Therefore, $f$ is transitive.

THEOREM 4.13. Let $X$ be a topological space, let $f: X \rightarrow X$ be a function and let $n \in \mathbb{N}$. If $\mathcal{F}_{n}(f)$ is weakly mixing, then $f$ is weakly mixing.

Proof. Suppose that $\mathcal{F}_{n}(f)$ is weakly mixing, we prove that $f$ is weakly mixing. Let $U_{1}, U_{2}, V_{1}$ and $V_{2}$ be nonempty open subsets of $X$. Then $\left\langle U_{1}\right\rangle_{n},\left\langle U_{2}\right\rangle_{n},\left\langle V_{1}\right\rangle_{n}$ and $\left\langle V_{2}\right\rangle_{n}$ are nonempty open subsets of $\mathcal{F}_{n}(X)$. Since $\mathcal{F}_{n}(f)$ is weakly mixing, there exists $k \in \mathbb{N}$ such that $\left[\mathcal{F}_{n}(f)\right]^{k}\left(\left\langle U_{i}\right\rangle_{n}\right) \cap\left\langle V_{i}\right\rangle_{n} \neq \varnothing$, for each $i \in\{1,2\}$. Then there exist elements $B_{1} \in\left\langle U_{1}\right\rangle_{n}$ and $B_{2} \in\left\langle U_{2}\right\rangle_{n}$ such that $\left[\mathcal{F}_{n}(f)\right]^{k}\left(B_{1}\right) \in\left\langle V_{1}\right\rangle_{n}$ and $\left[\mathcal{F}_{n}(f)\right]^{k}\left(B_{2}\right) \in\left\langle V_{2}\right\rangle_{n}$. Thus, we obtain that $f^{k}\left(B_{1}\right) \subseteq V_{1}$ and $f^{k}\left(B_{2}\right) \subseteq V_{2}$. Hence, $f^{k}\left(U_{1}\right) \cap V_{1} \neq \varnothing$, and $f^{k}\left(U_{2}\right) \cap V_{2} \neq \varnothing$. Therefore, $f$ is weakly mixing.

The following result is part of [21, Theorem 4.5].

THEOREM 4.14. Let $X$ be a topological space, let $f: X \rightarrow X$ be a function and let $n \in \mathbb{N}$. If $f$ is a map, then the following statements are equivalent:

(1) $f$ is weakly mixing.

(2) $\mathcal{F}_{n}(f)$ is weakly mixing.

(3) $\mathcal{F}_{n}(f)$ is transitive.

THEOREM 4.15. (compare with [7, Theorem 4.12]) Let $X$ be a topological space, let $f: X \rightarrow X$ be a function and let $n \in \mathbb{N}$. If $\mathcal{F}_{n}(X)$ is totally transitive, then $f$ is totally transitive.

Proof. Suppose that $\mathcal{F}_{n}(f)$ is totally transitive, we see that $f$ is totally transitive. Let $s \in \mathbb{N}$. We prove that $f^{s}$ is transitive. Let $U$ and $V$ be nonempty open subsets of $X$. Then $\langle U\rangle_{n}$ and $\langle V\rangle_{n}$ are nonempty open subsets of $\mathcal{F}_{n}(X)$. Since $\mathcal{F}_{n}(f)$ is totally transitive, $\left[\mathcal{F}_{n}(f)\right]^{s}$ is transitive. Thus, there exists $k \in \mathbb{N}$ such that $\left(\left[\mathcal{F}_{n}(f)\right]^{s}\right)^{k}\left(\langle U\rangle_{n}\right) \cap\langle V\rangle_{n} \neq \varnothing$; i.e., $\left[\mathcal{F}_{n}(f)\right]^{s k}\left(\langle U\rangle_{n}\right) \cap\langle V\rangle_{n} \neq \varnothing$. Then there exists $B \in\langle U\rangle_{n}$ such that $\left[\mathcal{F}_{n}(f)\right]^{s k}(B) \in\langle V\rangle_{n}$. Hence, $f^{s k}(B) \subseteq V$. Thus, $\left(f^{s}\right)^{k}(U) \cap V \neq \varnothing$. Therefore, $f^{s}$ is transitive. Since $s$ is an arbitrary positive integer, $f$ is totally transitive.

THEOREM 4.16. (compare with [7, Theorem 4.13]) Let $X$ be a topological space, let $f: X \rightarrow X$ be a function and let $n \in \mathbb{N}$. If $\mathcal{F}_{n}(f)$ is strongly transitive, then $f$ is strongly transitive.

Proof. Suppose that $\mathcal{F}_{n}(f)$ is strongly transitive, we show that $f$ is strongly transitive. Let $U$ be a nonempty open subset of $X$. Then $\langle U\rangle_{n}$ is a nonempty open subset of $\mathcal{F}_{n}(X)$. Thus, by hypothesis, there exists $s \in \mathbb{N}$ such that:

$$
\mathcal{F}_{n}(X)=\bigcup_{k=0}^{s}\left[\mathcal{F}_{n}(f)\right]^{k}\left(\langle U\rangle_{n}\right) .
$$

We prove that $X=\bigcup_{k=0}^{s} f^{k}(U)$. Let $x \in X$. We prove that $x \in \bigcup_{k=0}^{s} f^{k}(U)$. Note that $\{x\} \in \mathcal{F}_{n}(X)$. Thus, $\{x\} \in \bigcup_{k=0}^{s}\left[\mathcal{F}_{n}(f)\right]^{k}\left(\langle U\rangle_{n}\right)$. Then there exists $k_{0} \in\{0, \ldots, s\}$ such that $\{x\} \in\left[\mathcal{F}_{n}(f)\right]^{k_{0}}\left(\langle U\rangle_{n}\right)$. Hence, there exists $A \in\langle U\rangle_{n}$ such that $\left[\mathcal{F}_{n}(f)\right]^{k_{0}}(A)=\{x\}$. Since $A \subseteq U, x \in \bigcup_{k=0}^{s} f^{k}(U)$. Thus, $X \subseteq \bigcup_{k=0}^{s} f^{k}(U)$. Therefore, $f$ is strongly transitive.

THEOREM 4.17. Let $X$ be a topological space, let $f: X \rightarrow X$ be a function, let $n \in \mathbb{N}$ and let $A \in \mathcal{F}_{n}(X)$. If $A \in \operatorname{trans}\left(\mathcal{F}_{n}(f)\right)$, then, for each $a \in A, a \in \operatorname{trans}(f)$. 
Proof. Let $A \in \mathcal{F}_{n}(X)$ be such that $A \in \operatorname{trans}\left(\mathcal{F}_{n}(f)\right)$. Let $a \in A$. We show that $a \in \operatorname{trans}(f)$. Let $U$ be a nonempty open subset of $X$. Then $\langle U\rangle_{n}$ is a nonempty open subset of $\mathcal{F}_{n}(X)$ such that $\{a\} \in\langle U\rangle_{n}$. Since $A \in \operatorname{trans}\left(\mathcal{F}_{n}(f)\right), \operatorname{cl}_{\mathcal{F}_{n}(X)}\left(\mathcal{O}\left(A, \mathcal{F}_{n}(f)\right)\right)=\mathcal{F}_{n}(X)$. Hence, $\langle U\rangle_{n} \cap \mathcal{O}\left(A, \mathcal{F}_{n}(f)\right) \neq \varnothing$. Thus, there exists $k \in \mathbb{Z}_{+}$such that $\left[\mathcal{F}_{n}(f)\right]^{k}(A) \in\langle U\rangle_{n}$; i.e., $f^{k}(A) \subseteq U$. In particular, $f^{k}(a) \in U$. Hence, $f^{k}(a) \in U \cap \mathcal{O}(a, f)$ and $U \cap \mathcal{O}(a, f) \neq \varnothing$. Thus, $\operatorname{cl}_{X}(\mathcal{O}(a, f))=X$. Therefore, for each $a \in A$, we have that $a \in \operatorname{trans}(f)$.

COROLLARY 4.18. Let $X$ be a topological space, let $f: X \rightarrow X$ be a function and let $n \in \mathbb{N}$. If $\operatorname{trans}\left(\mathcal{F}_{n}(f)\right)=\mathcal{F}_{n}(X)$, then $\operatorname{trans}(f)=X$.

Proof. Suppose that $\operatorname{trans}\left(\mathcal{F}_{n}(f)\right)=\mathcal{F}_{n}(X)$. Let $x \in X$. Then $\{x\} \in \mathcal{F}_{n}(X)$. Hence, $\{x\} \in \operatorname{trans}\left(\mathcal{F}_{n}(f)\right)$. Then, by Theorem 4.17, $x \in \operatorname{trans}(f)$. Therefore, $\operatorname{trans}(f)=X$.

As consequence from Theorem 4.18 we have the following result:

THEOREM 4.19. Let $X$ be a topological space, let $f: X \rightarrow X$ be a function and let $n \in \mathbb{N}$. If $\operatorname{trans}\left(\mathcal{F}_{n}(f)\right)=\mathcal{F}_{n}(X)$, then $f$ is minimal.

THEOREM 4.20. Let $X$ be a topological space, let $f: X \rightarrow X$ be a function and let $n \in \mathbb{N}$. If $\mathcal{F}_{n}(f)$ is minimal, then $f$ is minimal.

Proof. Suppose that $\mathcal{F}_{n}(f)$ is minimal, we prove that $f$ is minimal. Let $F$ be a nonempty, closed and +invariant subset of $X$. We show that $X=F$. Note that $\langle F\rangle_{n}$ is a nonempty closed subset of $\mathcal{F}_{n}(X)$ (Theorem 3.2, part (6)). Clearly, $\langle F\rangle_{n}$ is +invariant under $\mathcal{F}_{n}(f)$. Since $\mathcal{F}_{n}(f)$ is minimal, $\langle F\rangle_{n}=\mathcal{F}_{n}(X)$. Let $x \in X$. Then $\{x\} \in \mathcal{F}_{n}(X)$. Thus, $\{x\} \in\langle F\rangle_{n}$. Hence, $x \in F$ and $X \subseteq F$. Therefore, $X=F$ and $f$ is minimal.

The following result extends the corresponding part of [21, Theorem 4.9] to arbitrary topological spaces and not necessarily continuous functions.

THEOREM 4.21. Let $X$ be a topological space, let $f: X \rightarrow X$ be a function and let $n \in \mathbb{N}$. If $\mathcal{F}_{n}(f)$ is chaotic, then $f$ is chaotic.

Proof. Suppose that $\mathcal{F}_{n}(f)$ is chaotic, we show that $f$ is chaotic. Since $\mathcal{F}_{n}(f)$ is chaotic, $\mathcal{F}_{n}(f)$ is transitive and $\operatorname{Per}\left(\mathcal{F}_{n}(f)\right)$ is dense in $\mathcal{F}_{n}(X)$. Then, by Theorem 4.12, we have that $f$ is transitive. Also, by Theorem 3.6, we obtain that $\operatorname{Per}(f)$ is a dense subset of $X$. Therefore, $f$ is chaotic.

In [21, Example 4.10], it is shown that the map $f$ of Example 4.22 is chaotic and the induced maps, $\mathcal{F}_{n}(f)$, are not chaotic, for any $n \geq 2$.

EXAMPLE 4.22. Let $X=[0,1]$ and $f: X \rightarrow X$ given by:

$$
f(x)= \begin{cases}2 x+\frac{1}{2}, & \text { if } x \in\left[0, \frac{1}{4}\right] \\ \frac{3}{2}-2 x, & \text { if } x \in\left[\frac{1}{4}, \frac{1}{2}\right] \\ 1-x, & \text { if } x \in\left[\frac{1}{2}, 1\right]\end{cases}
$$

The following theorem extends the corresponding part of [7, Theorem 5.6].

THEOREM 4.23. Let $X$ be a topological space, let $f: X \rightarrow X$ be a function and let $n \in \mathbb{N}$. If $f$ is turbulent, then $\mathcal{F}_{n}(f)$ is turbulent.

Proof. Suppose that $f$ is turbulent. Then, there exist compact nondegenerate subsets $C$ and $K$ of $X$ such that $C \cap K$ has at most one point and $K \cup C \subseteq f(K) \cap f(C)$. By Theorem 3.2, part (5), we have that $\langle C\rangle_{n}$ and $\langle K\rangle_{n}$ are compact subsets of $\mathcal{F}_{n}(X)$. Since $C \cap K \subset\{a\}$, it follows that $\langle C\rangle_{n} \cap\langle K\rangle_{n} \subset\{\{a\}\}$. Now, we show that $\langle C\rangle_{n} \cup\langle K\rangle_{n} \subseteq \mathcal{F}_{n}(f)\left(\langle K\rangle_{n}\right) \cap \mathcal{F}_{n}(f)\left(\langle C\rangle_{n}\right)$. Note that $\langle K\rangle_{n} \cup\langle C\rangle_{n} \subseteq\langle K \cup C\rangle_{n}$. Since $K \cup C \subseteq f(K) \cap f(C)$, we obtain that, $\langle K \cup C\rangle_{n} \subseteq\langle f(K) \cap f(C)\rangle_{n}$. Also, since $\langle f(K) \cap f(C)\rangle_{n}=\langle f(K)\rangle_{n} \cap\langle f(C)\rangle_{n}$ and $\langle f(K)\rangle_{n} \cap\langle f(C)\rangle_{n}=\left\langle\mathcal{F}_{n}(f)(K)\right\rangle_{n} \cap\left\langle\mathcal{F}_{n}(f)(C)\right\rangle_{n}$, we have that, $\langle K \cup C\rangle_{n} \subseteq\left\langle\mathcal{F}_{n}(f)(K)\right\rangle_{n} \cap\left\langle\mathcal{F}_{n}(f)(C)\right\rangle_{n}$. Hence, $\langle K \cup C\rangle_{n} \subseteq\left\langle\mathcal{F}_{n}(f)(K)\right\rangle_{n} \cap\left\langle\mathcal{F}_{n}(f)(C)\right\rangle_{n}$. Next, we prove that:

$$
\left\langle\mathcal{F}_{n}(f)(K)\right\rangle_{n} \cap\left\langle\mathcal{F}_{n}(f)(C)\right\rangle_{n} \subseteq \mathcal{F}_{n}(f)\left(\langle K\rangle_{n}\right) \cap \mathcal{F}_{n}(f)\left(\langle C\rangle_{n}\right) .
$$

Let $A \in\left\langle\mathcal{F}_{n}(f)(K)\right\rangle_{n} \cap\left\langle\mathcal{F}_{n}(f)(C)\right\rangle_{n}$. Then $A \subseteq f(K) \cap f(C)$. Thus, there exist $B \subseteq K$ and $D \subseteq C$ such that $f(B)=A$ and $f(D)=A$; i.e., $\mathcal{F}_{n}(f)(B)=A$ and $\mathcal{F}_{n}(f)(D)=A$. Hence, $A \in \mathcal{F}_{n}(f)\left(\langle K\rangle_{n}\right) \cap$ 
$\mathcal{F}_{n}(f)\left(\langle C\rangle_{n}\right)$. This proves our last claim. Therefore, we have that $\langle K\rangle_{n} \cup\langle C\rangle_{n} \subseteq \mathcal{F}_{n}(f)\left(\langle K\rangle_{n}\right) \cap$ $\mathcal{F}_{n}(f)\left(\langle C\rangle_{n}\right)$, and $\mathcal{F}_{n}(f)$ is turbulent.

QUESTION 4.24. Which conditions are needed to obtain the converse of Theorem 4.23?

The following result generalizes [7, Theorem 5.1] and its proof is essentially the same.

THEOREM 4.25. Let $X$ be a topological space, let $f: X \rightarrow X$ be a function and let $n \in \mathbb{N}$. If $\mathcal{F}_{n}(f)$ is irreducible, then $f$ is irreducible.

QUESTION 4.26. Which conditions are needed to obtain the converse of Theorem 4.25 ?

\section{OTHER TYPES OF TRANSITIVITY}

We consider the different types of transitivity that we define above.

THEOREM 5.1. Let $X$ be a topological space, let $f: X \rightarrow X$ be a function and let $n \in \mathbb{N}$. If $\mathcal{F}_{n}(f)$ is orbit-transitive, then $f$ is orbit-transitive.

Proof. Suppose that $\mathcal{F}_{n}(f)$ is orbit-transitive. We proved that $f$ is orbit-transitive. By hypothesis, there exists $A \in \mathcal{F}_{n}(X)$ such that $\operatorname{cl}_{\mathcal{F}_{n}(X)}\left(\mathcal{O}\left(A, \mathcal{F}_{n}(f)\right)\right)=\mathcal{F}_{n}(X)$. Hence, $A \in \operatorname{trans}\left(\mathcal{F}_{n}(f)\right)$. Let $a \in A$. By Theorem 4.17, $a \in \operatorname{trans}(f)$. Thus, $\operatorname{cl}_{X}(\mathcal{O}(a, f))=X$. Therefore, $f$ is orbit-transitive.

THEOREM 5.2. Let $X$ be a topological space, let $f: X \rightarrow X$ be a function and let $n \in \mathbb{N}$. If $f$ is orbit-transitive and each open subset of $X$ is +invariant under $f$, then $\mathcal{F}_{n}(f)$ is orbit-transitive.

Proof. Suppose that $f$ is orbit-transitive and that all the open subsets of $X$ are +invariant under $f$. Thus, there exists $x_{0} \in X$ with $\operatorname{cl}_{X}\left(\mathcal{O}\left(x_{0}, f\right)\right)=X$. We show that $\operatorname{cl}_{\mathcal{F}_{n}(X)}\left(\mathcal{O}\left(\left\{x_{0}\right\}, \mathcal{F}_{n}(f)\right)\right)=\mathcal{F}_{n}(X)$. Let $\mathcal{V}$ be a nonempty open subset of $\mathcal{F}_{n}(X)$. Then, there exist nonempty open subsets $U_{1}, \ldots, U_{r}$ of $X$ such that $\left\langle U_{1}, \ldots, U_{r}\right\rangle_{n} \subseteq \mathcal{V}$. Since $\operatorname{cl}_{X}(\mathcal{O}(x, f))=X$, for each $i \in\{1, \ldots, r\}$, there exists $k_{i} \in \mathbb{Z}_{+}$such that $f^{k_{i}}\left(x_{0}\right) \in U_{i}$. Let $k=\max \left\{k_{1}, \ldots, k_{r}\right\}$. Note that, for every $i \in\{1, \ldots, r\}, f^{k}\left(x_{0}\right)=f^{k-k_{i}}\left(f^{k_{i}}\left(x_{0}\right)\right)$. Since $f^{k_{i}}\left(x_{0}\right) \in U_{i}, f^{k-k_{i}}\left(f^{k_{i}}\left(x_{0}\right)\right) \in f^{k-k_{i}}\left(U_{i}\right)$. Because $U_{i}$ is +invariant, $f^{k-k_{i}}\left(f^{k_{i}}\left(x_{0}\right)\right) \in U_{i}$, for each $i \in\{1, \ldots, r\}$. Thus, for every $i \in\{1, \ldots, r\}, f^{k}\left(x_{0}\right) \in U_{i}$. Hence, $\left[\mathcal{F}_{n}(f)\right]^{k}\left(\left\{x_{0}\right\}\right) \in\left\langle U_{1}, \ldots, U_{r}\right\rangle_{n}$. Therefore, $\varnothing \neq\left\langle U_{1}, \ldots, U_{r}\right\rangle_{n} \cap \mathcal{O}\left(\left\{x_{0}\right\}, \mathcal{F}_{n}(f)\right) \subset \mathcal{V} \cap \mathcal{O}\left(\left\{x_{0}\right\}, \mathcal{F}_{n}(f)\right)$, and consequently $\mathcal{F}_{n}(f)$ is orbit-transitive.

THEOREM 5.3. Let $X$ be a topological space, let $f: X \rightarrow X$ be a function and let $n \in \mathbb{N}$. If $\mathcal{F}_{n}(f)$ is strictly orbit-transitive, then $f$ is strictly orbit-transitive.

Proof. Suppose that $\mathcal{F}_{n}(f)$ is strictly orbit-transitive. Then, there exists $\left\{x_{1}, \ldots, x_{r}\right\} \in \mathcal{F}_{n}(X)$ such that:

$$
\operatorname{cl}_{\mathcal{F}_{n}(X)}\left(\mathcal{O}\left(\mathcal{F}_{n}(f)\left(\left\{x_{1}, \ldots, x_{r}\right\}\right), \mathcal{F}_{n}(f)\right)\right)=\mathcal{F}_{n}(X) ;
$$

i.e., $\operatorname{cl}_{\mathcal{F}_{n}(X)}\left(\mathcal{O}\left(\left\{f\left(x_{1}\right), \ldots, f\left(x_{r}\right)\right\}, \mathcal{F}_{n}(f)\right)\right)=\mathcal{F}_{n}(X)$. By Theorem 4.17, we have that for each $i \in$ $\{1, \ldots, r\}, \operatorname{cl}_{X}\left(\mathcal{O}\left(f\left(x_{i}\right), f\right)\right)=X$. Therefore, $f$ is strictly orbit-transitive.

THEOREM 5.4. Let $X$ be a topological space, let $f: X \rightarrow X$ be a function and let $n \in \mathbb{N}$. If $f$ is strictly orbit-transitive and each open subset of $X$ is +invariant under $f$, then $\mathcal{F}_{n}(f)$ is strictly orbit-transitive.

Proof. Suppose that $f$ is strictly orbit-transitive and that all open subset of $X$ is +invariant under $f$. Then there exists $x_{0} \in X$ such that $\operatorname{cl}_{X}\left(\mathcal{O}\left(f\left(x_{0}\right), f\right)\right)=X$. Let $\mathcal{V}$ be a nonempty open subset of $\mathcal{F}_{n}(X)$. Hence, there exist nonempty open subsets $V_{1}, \ldots, V_{n}$ of $X$ such that $\left\langle V_{1}, \ldots V_{n}\right\rangle_{n} \subseteq \mathcal{V}$. By the election of $x_{0}$, for each $i \in\{1, \ldots, n\}$, there exists $k_{i} \in \mathbb{N}$ such that $f^{k_{i}}\left(x_{0}\right) \in V_{i}$. Let $k=\max \left\{k_{1}, \ldots, k_{n}\right\}$. By an argument similar to the one given in the proof of Theorem 5.3, we obtain that $\left\langle V_{1}, \ldots, V_{n}\right\rangle_{n} \cap$ $\mathcal{O}\left(\left\{f\left(x_{0}\right)\right\}, \mathcal{F}_{n}(f)\right) \neq \varnothing$. Therefore, $\mathcal{V} \cap \mathcal{O}\left(\left\{f\left(x_{0}\right)\right\}, \mathcal{F}_{n}(f)\right) \neq \varnothing$. Hence, $\operatorname{cl}_{\mathcal{F}_{n}(X)}\left(\mathcal{O}\left(\left\{f\left(x_{0}\right)\right\}, \mathcal{F}_{n}(f)\right)\right)=$ $\mathcal{F}_{n}(X)$. Thus, $\mathcal{F}_{n}(f)$ is strictly orbit-transitive.

THEOREM 5.5. Let $X$ be a topological space, let $f: X \rightarrow X$ be a function, let $n \in \mathbb{N}$ and let $A \in \mathcal{F}_{n}(X)$. If $\omega\left(A, \mathcal{F}_{n}(f)\right)=\mathcal{F}_{n}(X)$, then, for each $a \in A, \omega(a, f)=X$. 
Proof. Suppose that $\omega\left(A, \mathcal{F}_{n}(f)\right)=\mathcal{F}_{n}(X)$. Let $a \in A$. We see that $\omega(a, f)=X$. Let $y \in X$, let $k \in \mathbb{N}$ and let $U$ be an open subset of $X$ such that $y \in U$. Note that $\{y\} \in \mathcal{F}_{n}(X)$ and that $\langle U\rangle_{n}$ is an open subset of $\mathcal{F}_{n}(X)$ such that $\{y\} \in\langle U\rangle_{n}$. Since $\{y\} \in \omega\left(A, \mathcal{F}_{n}(f)\right)$, there exists $m>k$ such that $\left[\mathcal{F}_{n}(f)\right]^{m}(A) \in\langle U\rangle_{n}$. Hence, $f^{m}(A) \subseteq U$. Then $f^{m}(a) \in U$. Thus, $y \in \omega(a, f)$. Therefore, $\omega(a, f)=X$, for every $a \in A$.

As a consequence from Theorem 5.5, we have the following:

THEOREM 5.6. Let $X$ be a topological space, let $f: X \rightarrow X$ be a function and let $n \in \mathbb{N}$. If $\mathcal{F}_{n}(f)$ is $\omega$-transitive, then $f$ is $\omega$-transitive.

THEOREM 5.7. Let $X$ be a topological space, let $f: X \rightarrow X$ be a function and let $n \in \mathbb{N}$. If $f$ is $\omega$-transitive and each open subset of $X$ is +invariant, then $\mathcal{F}_{n}(f)$ is $\omega$-transitive.

Proof. Suppose that $f$ is $\omega$-transitive and that each open subset $U$ of $X$ is +invariant under $f$. Then there exists $x_{0} \in X$ such that $\omega\left(x_{0}, f\right)=X$. We prove that $\omega\left(\left\{x_{0}\right\}, \mathcal{F}_{n}(f)\right)=\mathcal{F}_{n}(X)$. Let $A \in \mathcal{F}_{n}(X)$, let $k \in \mathbb{N}$ and let $\mathcal{V}$ be an open subset of $\mathcal{F}_{n}(X)$ such that $A \in \mathcal{V}$. Then, there exist nonempty open subsets $V_{1}, \ldots, V_{n}$ of $X$ such that $A \in\left\langle V_{1}, \ldots, V_{n}\right\rangle_{n} \subseteq \mathcal{V}$. By election of $x_{0}$, for each $i \in\{1, \ldots, n\}$, there exists $m_{i} \geq k$ such that $f^{m_{i}}\left(x_{0}\right) \in V_{i}$. Let $m=\max \left\{m_{1}, \ldots, m_{n}\right\}$. Since $f^{n_{i}}\left(x_{0}\right) \in V_{i}$, we have that $f^{m-n_{i}}\left(f^{n_{i}}\left(x_{0}\right)\right) \in f^{m-n_{i}}\left(V_{i}\right)$. Because $V_{i}$ is +invariant under $f, f^{m-n_{i}}\left(V_{i}\right) \subseteq V_{i}$. Then, for every $i \in\{1, \ldots, n\}, f^{m}\left(x_{0}\right) \in V_{i}$. Hence, $\left[\mathcal{F}_{n}(f)\right]^{m}\left(\left\{x_{0}\right\}\right) \in\left\langle V_{1}, \ldots, V_{n}\right\rangle_{n} \subseteq \mathcal{V}$. Thus, $A \in \omega\left(\left\{x_{0}\right\}, \mathcal{F}_{n}(f)\right)$. Therefore, $\omega\left(\left\{x_{0}\right\}, \mathcal{F}_{n}(f)\right)=\mathcal{F}_{n}(X)$, and $\mathcal{F}_{n}(f)$ is $\omega$-transitive.

THEOREM 5.8. Let $X$ be a topological space, let $f: X \rightarrow X$ be a function and let $n \in \mathbb{N}$. If $\mathcal{F}_{n}(f)$ is $I N$, then $f$ is $I N$.

Proof. Suppose that $f$ is not $I N$. Then there exist two proper, closed and +invariant subsets $C_{1}$ and $C_{2}$ of $X$ such that $X=C_{1} \cup C_{2}$. By Theorem 3.2 part (3), $\left\langle X, C_{1}\right\rangle_{n}$ and $\left\langle X, C_{2}\right\rangle_{n}$ are closed subsets of $\mathcal{F}_{n}(X)$.

We show that, $\left\langle X, C_{1}\right\rangle_{n}$ and $\left\langle X, C_{2}\right\rangle_{n}$ are +invariant subsets under $\mathcal{F}_{n}(f)$ of $\mathcal{F}_{n}(X)$. Let $A \in$ $\left\langle X, C_{1}\right\rangle_{n}$. Then $A \cap C_{1} \neq \varnothing$. Since $f\left(A \cap C_{1}\right) \subseteq f(A) \cap f\left(C_{1}\right) \subseteq f(A) \cap C_{1}$, we obtain that $f(A) \cap C_{1} \neq \varnothing$. Hence, $f(A) \in\left\langle X, C_{1}\right\rangle_{n}$. Thus, $\mathcal{F}_{n}(f)\left(\left\langle X, C_{1}\right\rangle_{n}\right) \subseteq\left\langle X, C_{1}\right\rangle_{n}$, and $\left\langle X, C_{1}\right\rangle_{n}$ is +invariant under $\mathcal{F}_{n}(f)$. Similarly, we obtain that $\left\langle X, C_{2}\right\rangle_{n}$ is +invariant under $\mathcal{F}_{n}(f)$.

Clearly, $\left\langle X, C_{1}\right\rangle_{n}$ and $\left\langle X, C_{2}\right\rangle_{n}$ are proper subsets of $\mathcal{F}_{n}(X)$ and $\mathcal{F}_{n}(X)=\left\langle X, C_{1}\right\rangle_{n} \cup\left\langle X, C_{2}\right\rangle_{n}$. Therefore, $\mathcal{F}_{n}(f)$ is not $I N$.

THEOREM 5.9. Let $X$ be a topological space, let $f: X \rightarrow X$ be a function and let $n \in \mathbb{N}$. If $\mathcal{F}_{n}(f)$ is $T T_{++}$, then $f$ is $T T_{++}$.

Proof. Suppose that for each pair of nonempty open subsets $\mathcal{V}$ and $\mathcal{V}$ of $\mathcal{F}_{n}(X)$, the set $n_{\mathcal{F}_{n}(f)}(\mathcal{V}, \mathcal{V})$ is infinite. Let $U$ and $V$ be nonempty open subsets of $X$. Then $\langle U\rangle_{n}$ and $\langle V\rangle_{n}$ are open subsets of $\mathcal{F}_{n}(X)$. Since $\mathcal{F}_{n}(f)$ is $T T_{++}$, the set $n_{\mathcal{F}_{n}(f)}\left(\langle U\rangle_{n},\langle V\rangle_{n}\right)$ is infinite. We prove that $n_{\mathcal{F}_{n}(f)}\left(\langle U\rangle_{n},\langle V\rangle_{n}\right) \subseteq$ $n_{f}(U, V)$. Let $k \in n_{\mathcal{F}_{n}(f)}\left(\langle U\rangle_{n},\langle V\rangle_{n}\right)$. Then $\langle U\rangle_{n} \cap\left[\mathcal{F}_{n}(f)\right]^{-k}\left(\langle V\rangle_{n}\right) \neq \varnothing$. Let

$$
A \in\langle U\rangle_{n} \cap\left[\mathcal{F}_{n}(f)\right]^{-k}\left(\langle V\rangle_{n}\right) \text {. }
$$

Then $A \subseteq U$ and $\left[\mathcal{F}_{n}(f)\right]^{k}(A) \in\langle V\rangle_{n}$. Thus, $A \subseteq U$ and $A \subseteq f^{-k}(V)$. Hence, $U \cap f^{-k}(V) \neq \varnothing$. Thus, $k \in n_{f}(U, V)$, and $n_{\mathcal{F}_{n}(f)}\left(\langle U\rangle_{n},\langle V\rangle_{n}\right) \subseteq n_{f}(U, V)$. Therefore, $f$ satisfies the property $T T_{++}$.

THEOREM 5.10. Let $X$ be a topological space, let $f: X \rightarrow X$ be a function and let $n \in \mathbb{N}$. If $\mathcal{F}_{n}(f)$ is $\mathbb{Z}$-transitive, then $f$ is $\mathbb{Z}$-transitive.

Proof. Let $U$ and $V$ be nonempty open subsets of $X$. Note that $\langle U\rangle_{n}$ and $\langle V\rangle_{n}$ are nonempty open subsets of $\mathcal{F}_{n}(X)$. Since $\mathcal{F}_{n}(f)$ is $\mathbb{Z}$-transitive, it follows that $N_{\mathcal{F}_{n}(f)}\left(\langle U\rangle_{n},\langle V\rangle_{n}\right) \neq \varnothing$. Let $k \in N_{\mathcal{F}_{n}(f)}\left(\langle U\rangle_{n},\langle V\rangle_{n}\right)$. Then $\langle U\rangle_{n} \cap\left[\mathcal{F}_{n}(f)\right]^{-k}\left(\langle V\rangle_{n}\right) \neq \varnothing$. Let $A \in\langle U\rangle_{n} \cap\left[\mathcal{F}_{n}(f)\right]^{-k}\left(\langle V\rangle_{n}\right)$. Thus, $A \in\langle U\rangle_{n}$ and $\left[\mathcal{F}_{n}(f)\right]^{k}(A) \in\langle V\rangle_{n}$. Hence, $A \subset U$ and $f^{k}(A) \subset V$. This implies that $U \cap f^{-k}(V) \neq \varnothing$, and $k \in N_{f}(U, V)$. Therefore, $f$ is $\mathbb{Z}$-transitive.

A similar proof given in Theorem 5.10 gives:

THEOREM 5.11. Let $X$ be a topological space, let $f: X \rightarrow X$ be a function and let $n \in \mathbb{N}$. If $\mathcal{F}_{n}(f)$ is $\mathbb{Z}_{+}$-transitive, then $f$ is $\mathbb{Z}_{+}$-transitive. 
THEOREM 5.12. Let $X$ be a topological space, let $f: X \rightarrow X$ be a function and let $n \in \mathbb{N}$. If $\operatorname{trans}\left(\mathcal{F}_{n}(f)\right)$ is a dense subset in $\mathcal{F}_{n}(X)$, then $\operatorname{trans}(f)$ is a dense subset in $X$.

Proof. Suppose that $\operatorname{trans}\left(\mathcal{F}_{n}(f)\right)$ is a dense subset in $\mathcal{F}_{n}(X)$. We see that $\operatorname{trans}(f)$ is a dense subset in $X$. Let $U$ be a nonempty open subset of $X$. Then $\langle U\rangle_{n}$ is a nonempty open subset of $\mathcal{F}_{n}(X)$. Since $\operatorname{trans}\left(\mathcal{F}_{n}(f)\right)$ is dense in $\mathcal{F}_{n}(X),\langle U\rangle_{n} \cap\left(\operatorname{trans}\left(\mathcal{F}_{n}(f)\right)\right) \neq \varnothing$. Let $A \in\langle U\rangle_{n} \cap\left(\operatorname{trans}\left(\mathcal{F}_{n}(f)\right)\right) \neq \varnothing$. Hence, $A \subseteq U$ and $A \in \operatorname{trans}\left(\mathcal{F}_{n}(f)\right)$. Then, by Theorem 4.17, we have that $A \subset \operatorname{trans}(f)$. Thus, $A \subset U \cap \operatorname{trans}(f)$. Hence, $U \cap \operatorname{trans}(f) \neq \varnothing$, and $\operatorname{cl}_{X}(\operatorname{trans}(f))=X$. Therefore, $\operatorname{trans}(f)$ is a dense subset in $X$.

\section{RESULTS CONDITIONING THE FUNCTIONS AND/OR THE PHASE SPACES}

We begin with the following result, which is a consequence of [36, Theorem 5.10].

THEOREM 6.1. Let $X$ be a topological space and let $n \in \mathbb{N}$. A function $f: X \rightarrow X$ is continuous if and only if $\mathcal{F}_{n}(f): \mathcal{F}_{n}(X) \rightarrow \mathcal{F}_{n}(X)$ is continuous.

The following theorem is easy to establish.

THEOREM 6.2. Let $X$ be a topological space, let $f: X \rightarrow X$ be a function and let $n \in \mathbb{N}$.

a) $f$ is one-to-one if and only if $\mathcal{F}_{n}(f)$ is one-to-one;

b) $f$ is onto if and only if $\mathcal{F}_{n}(f)$ is onto.

THEOREM 6.3. Let $X$ be a topological space, let $f: X \rightarrow X$ be a function, let $n \in \mathbb{N}$ and let $A \in \mathcal{F}_{n}(X)$. Consider the following statements:

(a) $A \in \operatorname{trans}\left(\mathcal{F}_{n}(f)\right)$;

(b) For each $k \in \mathbb{N},\left[\mathcal{F}_{n}(f)\right]^{k}(A) \in \operatorname{trans}\left(\mathcal{F}_{n}(f)\right)$;

(c) For each $a \in A, a \in \operatorname{trans}(f)$;

(d) For each $a \in A$ and for every $k \in \mathbb{N}, f^{k}(a) \in \operatorname{trans}(f)$;

If $\mathcal{F}_{n}(X)$ does not have isolated points, then: (a) and (b) are equivalent; (c) and (d) are equivalent; (a) implies (c); and (b) implies (d).

Proof. Suppose that $\mathcal{F}_{n}(X)$ does not have quasi-isolated points. By [34, Lemma 2.6], we have that for each $k \in \mathbb{N}$ and every $A \in \mathcal{F}_{n}(X), A$ is a transitive point of $\mathcal{F}_{n}(f)$, if and only if $\left[\mathcal{F}_{n}(f)\right]^{k}(A)$ is a transitive point of $\mathcal{F}_{n}(f)$. By Theorem 3.17, we have that $X$ does not have quasi-isolated points. Hence, by [34, Lemma 2.6], we have that (c) and (d) are equivalent. Now, by Theorem 4.17, (a) implies (c). Now, if $k \in \mathbb{N}$ and $A=\left\{a_{1}, \ldots, a_{r}\right\} \in \mathcal{F}_{n}(X)$, then $\left[\mathcal{F}_{n}(f)\right]^{k}(A)=\left\{f^{k}\left(a_{1}\right), \ldots, f^{k}\left(a_{r}\right)\right\}$. Thus, if $\left[\mathcal{F}_{n}(f)\right]^{k}(A)$ is a transitive point of $\mathcal{F}_{n}(f)$, then by Theorem 4.17 , we have that $f^{k}\left(x_{i}\right)$ is a transitive point of $f$, for each $i \in\{1, \ldots, r\}$. Therefore, (b) implies (d).

THEOREM 6.4. Let $X$ be a topological space, let $f: X \rightarrow X$ be a function and let $n \in \mathbb{N}$. Consider the following statements:

(1) $\mathcal{F}_{n}(f)$ is $\omega$-transitive;

(2) $\mathcal{F}_{n}(f)$ is strictly orbit-transitive;

(3) $\mathcal{F}_{n}(f)$ is orbit-transitive;

(4) $\mathcal{F}_{n}(f)$ is transitive;

(5) $f$ is $\omega$-transitive;

(6) $f$ is strictly orbit-transitive;

(7) $f$ is orbit-transitive;

(8) $f$ is transitive.

If $X$ is $T_{1}$ without quasi-isolated points, then: (1), (2) and (3) are equivalent; (5), (6) and (7) are equivalent; (1) implies (5), (2) implies (6), (3) implies (7), (4) implies (8), (3) implies (4) and (7) implies (8).

Proof. Suppose that $X$ is a $T_{1}$ topological space without quasi-isolated points. By Theorem 3.19, we have that $\mathcal{F}_{n}(X)$ does not have quasi-isolated points. Then, by [34, Proposition 2.7], (2) and (3) are equivalent. By [34, Proposition 3.2], (2) and (1) are equivalent. Thus, (1), (2) and (3) are equivalent. Similarly, we show that (5), (6) and (7) are equivalent. By Theorem 5.3, (2) implies (6). Also, by 
Theorem 5.1, (3) implies (7). By Theorem 4.12, (4) implies (8). Finally, by [34, Proposition 4.1], (3) implies (4) and (7) implies (8).

THEOREM 6.5. Let $X$ be a topological space, let $f: X \rightarrow X$ be a function and let $n \in \mathbb{N}$. Consider the following statements:

(1) $\mathcal{F}_{n}(f)$ is $\omega$-transitive;

(2) $\mathcal{F}_{n}(f)$ is strictly orbit-transitive;

(3) $\mathcal{F}_{n}(f)$ is transitive;

(4) $f$ is $\omega$-transitive;

(5) $f$ is strictly orbit-transitive;

(6) $f$ is transitive.

If $f$ is a map and $X$ is partially compact and pseudo-regular with a countable base, then: (1) and (2) are equivalent; (4) and (5) are equivalent; (1) implies (4), (2) implies (5), (3) implies (4), (2) implies (3), and (5) implies (6).

Proof. Suppose that $f$ is a map and $X$ is partially compact and pseudo-regular with a countable base. Then, by [34, Proposition 3.2], we have that (4) and (5) are equivalent. Now, by Theorem 6.1, $\mathcal{F}_{n}(f)$ is a map. Again, by [34, Proposition 3.2], (1) and (2) are equivalent. Next, by Theorem 5.6, (1) implies (4). By Theorem 5.3, (2) implies (5). Now, we show that (3) implies (4). Suppose that $\mathcal{F}_{n}(f)$ is transitive. By Theorems 3.7 and $3.13, \mathcal{F}_{n}(X)$ has a countable base and it is partially compact and pseudo-regular. Thus, by [34, Proposition 5.7], $\mathcal{F}_{n}(f)$ is $\omega$-transitive and by Theorem 5.6, $f$ is $\omega$-transitive. Hence, (3) implies (4). By [34, Proposition 5.2], (2) implies (3) and (5) implies (6).

THEOREM 6.6. Let $X$ be a topological space, let $f: X \rightarrow X$ be a function and let $n \in \mathbb{N}$. Consider the following statements:

(1) $f$ is strictly orbit-transitive;

(2) $f$ is transitive;

(3) $\mathcal{F}_{n}(f)$ is strictly orbit-transitive;

(4) $\mathcal{F}_{n}(f)$ is transitive;

(5) $f$ is weakly mixing;

(6) $\mathcal{F}_{n}(f)$ is weakly mixing.

If $f$ is a map, then: (4), (5) and (6) are equivalent; (1) implies (2), (3) implies (4), (3) implies (1) and (4) implies (2).

Proof. Suppose that $f$ is a map. Then, by [34, Proposition 5.2], (1) implies (2). By Theorem 6.1, $\mathcal{F}_{n}(f)$ is a map. Thus, by [34, Proposition 5.2], (3) implies (4). By Theorem 5.3, (3) implies (1). By Theorem 4.12, (4) implies (2). Now, by Theorem 4.14, we have that (4), (5) and (6) are equivalent. $\square$

THEOREM 6.7. Let $X$ be a topological space, let $f: X \rightarrow X$ be a function and let $n \in \mathbb{N}$. Consider the following statements:

(1) $f$ is $T T_{++}$;

(2) $f$ is transitive;

(3) $f$ is $\mathbb{Z}_{+}$-transitive;

(4) $f$ is $\mathbb{Z}$-transitive;

(5) $f$ is $I N$;

(6) $\mathcal{F}_{n}(f)$ is $T T_{++}$;

(7) $\mathcal{F}_{n}(f)$ is transitive;

(8) $\mathcal{F}_{n}(f)$ is $\mathbb{Z}_{+}$-transitive;

(9) $\mathcal{F}_{n}(f)$ is $\mathbb{Z}$-transitive;

(10) $\mathcal{F}_{n}(f)$ is $I N$.

If $X$ is a Hausdorff and perfect space and $f$ is a map, then: (1), (2), (3), (4) and (5) are equivalent; (6), (7), (8), (9) and(10) are equivalent; (6) implies (1), (7) implies (2), (8) implies (3), (9) implies (4) and (10) implies (5).

Proof. Suppose that $X$ is a perfect Hausdorff topological space and that $f$ is a map. By [2, Theorem 1.4], we have that (1), (2), (4) and (5) are equivalent. Then, by the diagram of Figure 1, we have that (1), (2), (3), (4) and (5) are equivalent. By Theorem 5.9, (6) implies (1), by Theorem 4.12, (7) implies (2). 
By Theorem 5.11, (8) implies (3). By Theorem 5.10, (9) implies (4) and by Theorem 5.8, (10) implies (5). Now, by Theorems 3.9, 3.16 and 6.1, we have that $\mathcal{F}_{n}(X)$ is a perfect and Hausdorff space, and $\mathcal{F}_{n}(f)$ is a map. Again, by [2, Theorem 1.4] and by the diagram in Figure 1, we have that (6), (7), (8), (9) and (10) are equivalent.

\section{REFERENCES}

[1] Aкın, E. The General Topology of Dynamical Systems, vol. 1. American Mathematical Society, Providence, 1993.

[2] Akin, E., ANd Carlson, J. Conceptions of topological transitivity. Topology and its Applications 159 (2012), 2815-2830. doi:10.1016/j.topol.2012.04.016.

[3] Auslander, J., Greschonig, G., And Nagar, A. Reflections on equicontinuity. Proceedings of the American Mathematical Society 142 (2014), 3129-3137. doi:10.1090/S0002-9939-2014-12034-X.

[4] BAnks, J. Topological mapping properties defined by digraphs. Discrete \& Continuous Dynamical Systems 5 (1999), 83-92. doi:10.3934/dcds.1999.5.83.

[5] BAnks, J. Chaos for induced hyperspace maps. Chaos, Solitons \& Fractals 25 (2005), 681-685. doi:10.1016/j.chaos.2004.11.089.

[6] Barragán, F., Macías, S., AND TEnorio, J. More on induced maps on $n$-fold symmetric product suspensions. Glasnik Matematicki 50 (2015), 489-512. doi:10.3336/gm.50.2.15.

[7] Barragán, F., Santiago, A., And Tenorio, J. F. Dynamic properties for the induced maps on $n$-fold symmetric product suspensions. Glasnik Matematicki 51 (2016), 453-474. doi:10.3336/gm.51.2.12.

[8] BAuER, W., AND Sigmund, K. Topological dynamics of transformations induced on the space of probability measures. Monatshefte für Mathematik 79 (1975), 81-92. doi:10.1007/BF01585664.

[9] Bilokopytov, E., ANd KolyadA, S. Transitive maps on topological spaces. Ukrainian Mathematical fournal 65 (2014), 1293-1318. doi:10.1007/s11253-014-0860-8.

[10] Birkhoff, G. Dynamical Systems, vol. 9. American Mathematical Society, Providence, 1927.

[11] Borsuk, K., AND Ulam, S. On symmetric products of topological space. Bulletin of the American Mathematical Society 37 (1931), 875-882. doi:10.1090/s0002-9904-1931-05290-3.

[12] Brown, T., ANd Comfort, W. New method for expansion and contraction maps in uniform spaces. Proceedings of the American Mathematical Society 11 (1960), 483-486. doi:10.2307/2034804.

[13] Bryant, B. On expansive homeomorphisms. Pacific fournal of Mathematics 10 (1960), 11631167.

[14] Fernández, L., Good, C., And M. Puljiz, Á. R. Chain transitivity in hyperspaces. Chaos, Solitons \& Fractals 81 (2015), 83-90. doi:10.1016/j.chaos.2015.08.026.

[15] Furstenberg, H. Disjointness in ergodic theory, minimal sets, and a problem in diophantine approximation. Mathematical systems theory 1 (1967), 1-49. doi:10.1007/BF01692494.

[16] Gómez, J., Illanes, A., AND MÉndez, H. Dynamic properties for the induced maps in the symmetric products. Chaos Solitons \& Fractals 45 (2012), 1180-1187. doi:10.1016/j.chaos.2012.05.003.

[17] Good, C., AND Macías, S. Symmetric products of generalized metric spaces. Topology and its Applications 206 (2016), 93-114. doi:10.1016/j.topol.2016.03.019.

[18] Good, C., AND Macías, S. What is topological about topological dynamics? Discrete \& Continuous Dynamical Systems - A 38 (2018), 1007-1031. doi:10.3934/dcds.2018043.

[19] Gotschalk, W., And Hedlun, G. Topological Dynamics, vol. 36. American Mathematical Society, Providence, 1955.

[20] Hausdorff, F. Grundzuge der Mengenlehre. Leipzig, 1914.

[21] Higuera, G., And Illanes, A. Induced mappings on symmetric products. Topology Proceedings 37 (2011), 367-401.

[22] Hoon, B. Topological entropy and uniform spaces. Fournal of the London Mathematical Society 8 (1974), 633-641. doi:10.1112/jlms/s2-8.4.633.

[23] Illanes, A., AND JR., S. N. Hyperspaces: fundamentals and recent advances, vol. 216 of Monographs and Textbooks in Pure and Applied Math. Marcel Dekker, New York, Basel, 1999.

[24] JR., S. N. Hyperspaces of sets, vol. 49 of Monographs and Textbooks in Pure and Applied Math. Marcel Dekker, New York, Basel, 1978. Reprinter in: Aportaciones Matemáticas, Serie Textos No. 33, Sociedad Matemática Mexicana, 2006. 
[25] Kolyada, S., Snoha, L., ANd Trofimchuk, S. Noninvertible minimal maps. Fundamenta Mathematicae 168 (2001), 141-163. doi:10.4064/fm168-2-5.

[26] Kwietniak, D., And Misiurewicz, M. Exact devaney chaos and entropy. Qualitative Theory of Dynamical Systems 6 (2005), 169-179. doi:10.1007/BF02972670.

[27] Kwietniak, D., AND Oprocha, P. Topological entropy and chaos for maps induced on hyperspaces. Chaos, Solitons \& Fractals 33 (2007), 76-86. doi:10.1016/j.chaos.2005.12.033.

[28] Lampart, M., AND Raith, P. Topological entropy for set valued maps. Nonlinear Analysis 73 (2010), 1533-1537. doi:10.1016/j.na.2010.04.054.

[29] LI, R. A note on stronger forms of sensitivity for dynamical systems. Chaos Solitons \& Fractals 45 (2012), 753-758. doi:10.1016/j.chaos.2012.02.003.

[30] LiaO, G., WANG, L., AND Zhang, Y. Transitivity, mixing and chaos for a class of set-valued mappings. Science in China Series A 49 (2006), 1-8. doi:10.1007/s11425-004-5234-5.

[31] Macías, S. Aposyndetic properties of symmetric product of continua. Topology Proceedings 22 (1997), 281-296.

[32] Macías, S. On symmetric product of continua. Topology and its Applications 92 (1999), 173-182. doi:10.1016/S0166-8641(97)00233-2.

[33] Macías, S. Topics on Continua, 2 ed. Springer, 2018.

[34] MAI, J., AND SUn, W. Transitivities of maps of general topological spaces. Topology and its Applications 157 (2010), 946-953. doi:10.1016/j.topol.2009.12.011.

[35] Martínez-Montejano, J. Mutual aposyndesis of symmetric products. Topology Proceedings 24 (1999).

[36] Michael, E. Topologies on spaces of subsets. Transactions of the American Mathematical Society 71 (1951), 152-182. doi:10.1090/S0002-9947-1951-0042109-4.

[37] Peña, J., And López, G. Topological entropy for induced hyperspace maps. Chaos Solitons \& Fractals 28 (2006), 979-982. doi:10.1016/j.chaos.2005.08.173.

[38] Peng, L., AND Sun, Y. A study of symmetric products of generalized metric spaces. Topology and its Applications 231 (2017), 411-429. doi:10.1016/j.topol.2017.09.036.

[39] Peris, A. Set-valued discrete chaos. Chaos, Solitons \& Fractals 26 (2005), 19-23. doi:10.1016/j.chaos.2004.12.039.

[40] Román-Flores, H. A note on transitivity in set-valued discrete systems. Chaos Solitons \& Fractals 17 (2003), 99-104. doi:10.1016/S0960-0779(02)00406-X.

[41] Sabbaghan, M., AND Damerchiloo, H. A note on periodic points and transitive maps. Mathematical Sciences 5 (2011), 259-266.

[42] TANG, Z., Lin, S., AND Lin, F. Symmetric products and closed finite-to-one mappings. Topology and its Applications 234 (2018), 26-45. doi:10.1016/j.topol.2017.11.004.

[43] ViEtoris, L. Bereiche zweiter ordnung. Monatshefte für Mathematik und Physik 32 (1922), 258-280. doi:10.1007/BF01696886.

[44] Wang, Y., WeI, G., AND CAmpbelL, W. Sensitive dependence on initial conditions between dynamical systems and their induced hyperspace dynamical systems. Topology and its Applications 156 (2009), 803-811. doi:10.1016/j.topol.2008.10.014.

Open Access statement. This is an open-access article distributed under the terms of the Creative Commons AttributionNonCommercial 4.0 International License (https://creativecommons.org/licenses/by-nc/4.0/), which permits unrestricted use, distribution, and reproduction in any medium for non-commercial purposes, provided the original author and source are credited, a link to the CC License is provided, and changes - if any - are indicated. 